\title{
Fusarium Consortium Populations Associated with Asparagus Crop in Spain and Their Role on Field Decline Syndrome
}

\author{
Alexandri María Brizuela ${ }^{1}$ (D), Eduardo De la Lastra ${ }^{2}$, José Ignacio Marín-Guirao ${ }^{3}$, \\ Laura Gálvez $^{1}$, Miguel de Cara-García ${ }^{3}$ (D) Nieves Capote ${ }^{2, *(D)}$ and Daniel Palmero ${ }^{1, *(D)}$ \\ 1 Department of Agricultural Production, Escuela Técnica Superior de Ingeniería Agronómica, \\ Alimentaria y de Biosistemas, Universidad Politécnica de Madrid, 28040 Madrid, Spain; \\ alexandri.brizuela@alumnos.upm.es (A.M.B.); laura.galvez@upm.es (L.G.) \\ 2 Institute for Research and Training in Agriculture and Fisheries, IFAPA Las Torres. Ctra. Sevilla, \\ Cazalla, Km. 12,2, Alcalá del Río, 41200 Sevilla, Spain; eduardo.lastra@juntadeandalucia.es \\ 3 Institute for Research and Training in Agriculture and Fisheries, IFAPA La Mojonera, Camino San Nicolás, 1. \\ La Mojonera, 04745 Almería, Spain; josei.marin@juntadeandalucia.es (J.I.M.-G.); \\ franciscom.cara@juntadeandalucia.es (M.d.C.-G.) \\ * Correspondence: marian.capote@juntadeandalucia.es (N.C.); daniel.palmero@upm.es (D.P.)
}

Academic Editors: José Díaz and Javier Veloso

Received: 14 October 2020; Accepted: 29 November 2020; Published: 4 December 2020

check for updates

\begin{abstract}
Asparagus Decline Syndrome (ADS) is one of the main phytosanitary problems of asparagus crop worldwide. Diseased plants and soil samples from 41 fields from three main production areas of Spain were surveyed. Eight Fusarium species belonging to seven species complexes were identified in soils: F. oxysporum, F. proliferatum, F. redolens, F. solani sensu stricto, F. equiseti, F. culmorum, F. compactum and F. acuminatum. Fusarium oxysporum was the most prevalent species. Statistical correlation $\left(\mathrm{R}^{2}=88 \%\right.$ ) was established between F. oxysporum inoculum density and the average temperature of the warmest month. A relationship was also established between three crop factors (average temperature, crop age and F. oxysporum inoculum density) and field disease indices. Significant differences were observed between the distribution of F. oxysporum propagules in white and green asparagus fields. Thirteen Fusarium species belonging to seven species complexes were identified from roots of diseased plants, being F. oxysporum the most prevalent. F. proliferatum, F. oxysporum and F. redolens showed pathogenicity to asparagus and were the main species associated to ADS. Fusarium oxysporum was the species with the highest genetic diversity displaying 14 sequence-based haplotypes with no geographic differentiation. This work contributes to understanding the Fusarium complex associated to ADS for developing accurate integrated disease management strategies.
\end{abstract}

Keywords: Asparagus Decline Syndrome (ADS); F. oxysporum f. sp. asparagi; F. proliferatum; F. redolens; field disease index; pathogenicity; genetic diversity

\section{Introduction}

Asparagus (Asparagus officinalis L.) is one of the most widely distributed open field horticultural crops in the European Union. With more than 14,688 ha dedicated to the crop and production of $68,403 \mathrm{t}$, Spain is the fifth largest producer in the world and the second largest in Europe after Germany, both among the five biggest world producers [1]. Exports are around 24,701 $\mathrm{t}$ with a total value of 70.4 million euros [2]. However, the crop has experienced a substantial yield decrease over the last few seasons due to fungal diseases.

Asparagus Decline Syndrome (ADS) is one of the main phytosanitary problems of asparagus crop worldwide [3]. It is characterized by a gradual loss of vigor which can even lead to the death of 
affected plants. The symptoms associated with the disease are variable and can be observed in the different phases of the crop, from the seedlings and small crowns used in the plantation to the adult plants in full production. In seedling stage, the main symptomatology is root rot that, in severe cases, can cause the death of seedlings [4]. In young crowns, reddish coloration and rots in the secondary root system are observed that begin from the tips and end with the complete disappearance of the secondary root [5]. Storage roots take longer to express symptoms, and brown spots appear on them, which in their early stages do not progress in depth. Later, the roots collapse and empty, leaving only the hollow epidermal cover. In adult plants, the pathogen progresses through the vascular tissues until it reaches the crown, where a cross section of the claw allows the observation of a dark brown rot. It is in older crops where symptoms are most clearly seen. After the productive period, when the plants are left to vegetate in order to store the necessary reserves for the following year, the affected plants begin to show a premature yellowing that could be confused with that caused by root asphyxia due to waterlogging. The most affected stems become completely dry and finally the whole plant wilts.

This is a complex syndrome in whose causality both abiotic, such as water stress or allelopathic compounds, and biotic factors could be involved [6,7], but in which the Fusarium complex plays a predominant role [8-10]. The syndrome is expressed by shortening the productive period of the crop and limiting the replanting of asparagus on soils previously cultivated with asparagus by leading to the phenomenon known as "soil fatigue" [11]. This phenomenon appears in the second and third year after replanting, which does not depend on the rest period between plantations, and is characterized by a marked decrease in the vigor of the plants combined with a smaller size of harvested sprouts. The decrease in the yield is so drastic that on many occasions it makes the crop unprofitable.

The disease was first described more than a century ago, but its biotic causal agent was not identified until 1941, when Cohen and Heald [12] described it as F. oxysporum f. sp. asparagi. Since then, different researchers have addressed the study of the disease by extending its etiology to a complex of Fusarium species that have been associated with the syndrome, including F. proliferatum, F. redolens, F. solani or F. ershadii, among others [9,13-19].

Among all of them, F. oxysporum f. sp. asparagi and F. proliferatum are the species that seem to prevail as the most pathogenic within the specific complex associated to the disease, although the composition of the fungal consortium is highly variable depending on the geographical region under study. ADS was first reported in Spain in the 1980s [20]. Several of the Fusarium species previously described in other areas of the world were also isolated in our country, particularly the most abundant species, F. oxysporum and F. proliferatum (sensu Messiaen and Cassini [21]), whose pathogenicity was demonstrated.

This disease is difficult to control due to the multiannual character of the asparagus crop, easy dissemination with the propagation material and the persistence of asparagus root residues and inoculum of the Fusaria consortium over time, recovering asparagus root residues with high contents of fusaric microbiota after 25 years without cultivation $[9,17]$. On the other hand, the available asparagus cultivars have low resistance [22-24]. Although there is a source of resistance to Fusarium in Asparagus densiflorus [25], it is difficult to obtain resistant cultivars under various agro-environmental conditions due to the high genetic and pathogenic diversity of the Fusaria consortium, whose species composition and preponderance varies with the climatic conditions. An accurate identification of the species associated to ADS is crucial for management. A multilocus sequence typing database, Fusarium MLST (http://www.westerdijkinstitute.nl/fusarium) with partial sequences from phylogenetically informative loci was developed to facilitate accurate identification of single phylogenetic Fusarium species. Several gene fragments can be amplified by PCR and sequenced using primers that are conserved across the phylogenetic breadth of Fusarium. The phylogenetic concept of species, based on DNA sequence homology and phylogenetic analysis, has allowed the description of multiple Fusarium species (originally associated to a single morphological species) belonging to different species complexes. In addition, estimating the genetic diversity and the population structure of Fusarium consortium associated to ADS in the three asparagus production areas can increase our understanding of the 
disease and improve the management. In this work, a multilocus sequence dataset was constructed consisting of concatenated partial sequences of genes for the translation elongation factor 1-alpha $(E F-1 \alpha)$ and the DNA-directed RNA polymerase II largest (RPB1) and second largest subunit (RPB2) to accurately identify and to assess the genetic diversity of the Fusarium consortium associated to asparagus in Spain.

The present study aimed to describe the role of Fusarium consortium on ADS by analyzing asparagus fields in the main Spanish production areas. To achieve this, Fusarium isolates were obtained from diseased asparagus plants and soils from the north, center and south of Spain, corresponding to Navarra, Madrid and Andalusia regions. Fusarium isolates were morphologically and molecularly identified via multilocus sequence typing, and the relative abundance of each species in the three production areas and their pathogenicity to asparagus were determined. In addition, the potential correlation between the climatic conditions of the cultivation area and the occurrence of Fusarium oxysporum soil inoculum density and the relationship between three crop parameters (average climate temperature, crop age and F. oxysporum soil inoculum density) and field disease index (FDI) values were estimated. Finally, the phylogenetic relationships among isolates and the genetic diversity of the pathogenic Fusarium species detected in the Spanish production areas were calculated.

\section{Materials and Methods}

\subsection{Asparagus Fields Sampling}

Asparagus is a broad distributed crop in Spain. Sampling was designed to cover not only different cultivated varieties and growing methods but also different climatic regions. In total, 41 fields from three provinces covering seven different municipalities were sampled for soil and plants. Soils from plots with different crop ages ( $0-22$ years) were sampled and analyzed. In total, 16 fields were collected in the north of the country (Navarra) (1-16), 6 in the center (Madrid) (17-22) and 20 in the south (Andalusia) (23-41). Sampling dates, climate and location of the sampled fields are summarized in Table 1 and Figure 1.

Sampling dates were October 2017 for fields from the north and center of the country and June and July 2018 for fields from the south. Samples from north and center were obtained during vegetative development after the harvest period. Disease Severity Index was determined in the field by two independent observers as percentage of prematurely yellowed plants. All percentage values for field disease index were arcsine square root transformed before statistical analysis. Samples from the south were collected during harvest period (Table 1). For each municipality, climatic data were collected from the regional basic climatological network.

All plant samples were manually collected and consisted in 4-6 well-developed plants per field (including the root system). Prematurely yellowed, dried and dead plants were selected for Fusarium spp. isolation. Within the plants, pieces of feeder and storage roots showing brown or necrotic spots, loss of feeder roots, root collapse and/or root rot were taken. Soil samples $(3 \mathrm{~L})$ were collected surrounding the roots at a depth of 5-30 cm from the root zone, using a disinfected drill. After collecting, samples were taken to the laboratory for analysis within $24-48 \mathrm{~h}$. 
Table 1. Samples codes, origin, climate, varieties, cultivation type and sowing date.

\begin{tabular}{|c|c|c|c|c|c|c|}
\hline Sample & Region & Municipality & Variety & $\begin{array}{l}\text { Cultivation } \\
\text { Type }\end{array}$ & $\begin{array}{c}\text { Köppen-Geiger } \\
\text { Climate Classification } \\
\text { System [26] }\end{array}$ & Sowing Date \\
\hline 1 & Navarra & Ancín & Grolim & White asparagus & Oceanic (Cfb) & 2012 \\
\hline 2 & Navarra & Ancín & Grolim & White asparagus & Oceanic (Cfb) & 2017 \\
\hline 3 & Navarra & Ancín & Grolim & White asparagus & Oceanic (Cfb) & 2009 \\
\hline 4 & Navarra & Ancín & Grolim & White asparagus & Oceanic (Cfb) & 2012 \\
\hline 5 & Navarra & Ancín & Grolim & White asparagus & Oceanic (Cfb) & 2017 \\
\hline 6 & Navarra & Ancín & Fortens & White asparagus & Oceanic (Cfb) & 2016 \\
\hline 7 & Navarra & Ancín & Grolim & White asparagus & Oceanic ( $\mathrm{Cfb})$ & 2014 \\
\hline 8 & Navarra & Ancín & Grolim & White asparagus & Oceanic (Cfb) & 2016 \\
\hline 9 & Navarra & Ancín & Cygnus & White asparagus & Oceanic $(\mathrm{Cfb})$ & 2016 \\
\hline 10 & Navarra & Larraga & Grolim & White asparagus & Oceanic (Cfb) & 2009 \\
\hline 11 & Navarra & Berbinzana & Grolim & White asparagus & Oceanic (Cfb) & 2009 \\
\hline 12 & Navarra & Berbinzana & Grolim & White asparagus & Oceanic (Cfb) & 2009 \\
\hline 13 & Navarra & $\begin{array}{l}\text { Miranda } \\
\text { de Arga }\end{array}$ & Grolim & White asparagus & Oceanic (Cfb) & 2010 \\
\hline 14 & Navarra & $\begin{array}{l}\text { Miranda } \\
\text { de Arga }\end{array}$ & Grolim & White asparagus & Oceanic (Cfb) & 2012 \\
\hline 15 & Navarra & Tudela & Grolim & White asparagus & $\begin{array}{c}\text { Warm-summer } \\
\text { Mediterranean (Csb) }\end{array}$ & 2006 \\
\hline 16 & Navarra & Tudela & Grolim & White asparagus & $\begin{array}{c}\text { Warm-summer } \\
\text { Mediterranean (Csb) }\end{array}$ & 2014 \\
\hline 17 & Madrid & Aranjuez & Grande & Green asparagus & Cold semi-arid (BSk) & 2011 \\
\hline 18 & Madrid & Aranjuez & Grande & Green asparagus & Cold semi-arid (BSk) & 2017 \\
\hline 19 & Madrid & Aranjuez & Grande & Green asparagus & Cold semi-arid (BSk) & 2012 \\
\hline 20 & Madrid & Aranjuez & Grande & Green asparagus & Cold semi-arid (BSk) & 2011 \\
\hline 21 & Madrid & Aranjuez & Grande & Green asparagus & Cold semi-arid (BSk) & 2014 \\
\hline 22 & Madrid & Aranjuez & Grande & Green asparagus & Cold semi-arid (BSk) & 2010 \\
\hline 23 & Andalusia & Huétor-Tájar & Grande & Green asparagus & $\begin{array}{c}\text { Hot-summer } \\
\text { Mediterranean (Csa) }\end{array}$ & 2010 \\
\hline 24 & Andalusia & Huétor-Tájar & Grande & Green asparagus & $\begin{array}{c}\text { Hot-summer } \\
\text { Mediterranean (Csa) }\end{array}$ & 2012 \\
\hline 25 & Andalusia & Huétor-Tájar & Grande & Green asparagus & $\begin{array}{c}\text { Hot-summer } \\
\text { Mediterranean (Csa) }\end{array}$ & 2012 \\
\hline 26 & Andalusia & Huétor-Tájar & Grande & Green asparagus & $\begin{array}{c}\text { Hot-summer } \\
\text { Mediterranean (Csa) }\end{array}$ & 1995 \\
\hline 27 & Andalusia & Huétor-Tájar & Grande & Green asparagus & $\begin{array}{c}\text { Hot-summer } \\
\text { Mediterranean (Csa) }\end{array}$ & 2016 \\
\hline 28 & Andalusia & Huétor-Tájar & Grande & Green asparagus & $\begin{array}{c}\text { Hot-summer } \\
\text { Mediterranean (Csa) }\end{array}$ & 2015 \\
\hline 29 & Andalusia & Loja & Grande & Green asparagus & $\begin{array}{c}\text { Hot-summer } \\
\text { Mediterranean (Csa) }\end{array}$ & 2013 \\
\hline 30 & Andalusia & Loja & Grande & Green asparagus & $\begin{array}{c}\text { Hot-summer } \\
\text { Mediterranean (Csa) }\end{array}$ & 2014 \\
\hline 31 & Andalusia & Loja & Grande & Green asparagus & $\begin{array}{c}\text { Hot-summer } \\
\text { Mediterranean (Csa) }\end{array}$ & 2011 \\
\hline 32 & Andalusia & Loja & Atlas & Green asparagus & $\begin{array}{c}\text { Hot-summer } \\
\text { Mediterranean (Csa) }\end{array}$ & 2016 \\
\hline 33 & Andalusia & Loja & Grande & Green asparagus & $\begin{array}{c}\text { Hot-summer } \\
\text { Mediterranean (Csa) }\end{array}$ & 2012 \\
\hline 34 & Andalusia & Loja & Grande & Green asparagus & $\begin{array}{c}\text { Hot-summer } \\
\text { Mediterranean (Csa) }\end{array}$ & 2005 \\
\hline 35 & Andalusia & Loja & Grande & Green asparagus & $\begin{array}{c}\text { Hot-summer } \\
\text { Mediterranean (Csa) }\end{array}$ & 2010 \\
\hline 36 & Andalusia & Loja & Grande & Green asparagus & $\begin{array}{c}\text { Hot-summer } \\
\text { Mediterranean (Csa) }\end{array}$ & 2013 \\
\hline 37 & Andalusia & Loja & Placosesp & Green asparagus & $\begin{array}{c}\text { Hot-summer } \\
\text { Mediterranean (Csa) }\end{array}$ & 2012 \\
\hline 38 & Andalusia & Loja & Placosesp & Green asparagus & $\begin{array}{c}\text { Hot-summer } \\
\text { Mediterranean (Csa) }\end{array}$ & 2008 \\
\hline 39 & Andalusia & El Jau & Grande & Green asparagus & $\begin{array}{c}\text { Hot-summer } \\
\text { Mediterranean (Csa) }\end{array}$ & 2010 \\
\hline 40 & Andalusia & El Jau & Grande & Green asparagus & $\begin{array}{c}\text { Hot-summer } \\
\text { Mediterranean (Csa) }\end{array}$ & 2010 \\
\hline 41 & Andalusia & El Jau & Grande & Green asparagus & $\begin{array}{c}\text { Hot-summer } \\
\text { Mediterranean (Csa) }\end{array}$ & 2010 \\
\hline
\end{tabular}




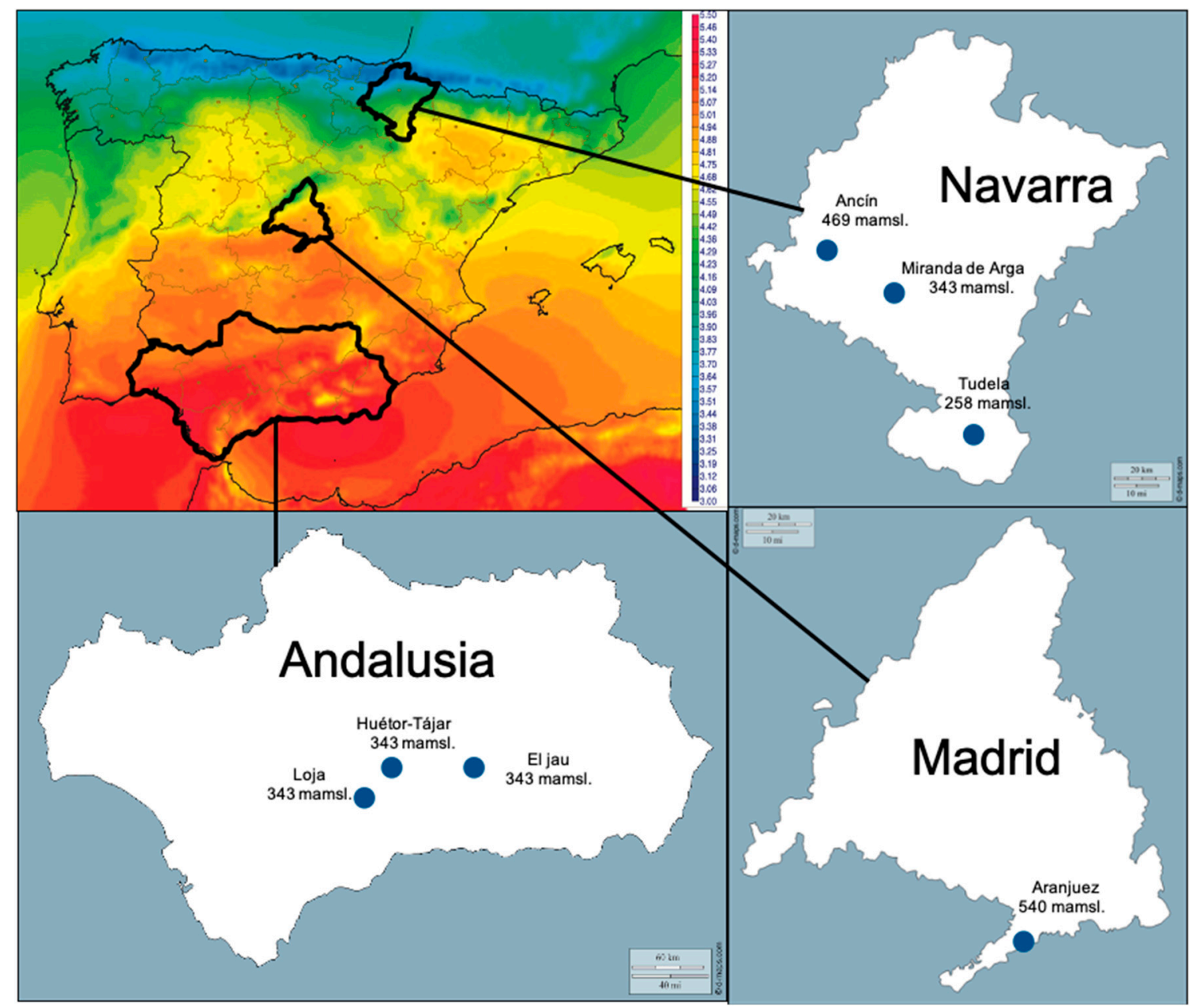

Figure 1. Surface Incoming Radiation map - AEMET (1983-2005) ( $\mathrm{kwh} \mathrm{m}^{-2} \mathrm{day}^{-1}$ ) (Climate-Satellite Application Facilities), location and altitude of sampled municipalities.

\subsection{Analysis of Plant Samples}

Analysis of the plant samples consisted in the superficial disinfection of secondary and storing roots with $1.5 \%$ sodium hypochlorite solution for $1 \mathrm{~min}$, followed by two successive washings with sterile distilled water. After drying, $1 \mathrm{~cm}$ pieces were sown in plates with potato dextrose agar (PDA) culture medium supplemented with $0.5 \mathrm{~g} / \mathrm{L}$ of streptomycin sulphate (Sigma-Aldrich, St. Louis, MO, USA) (PDAS) and incubated for 5-7 days at laboratory temperature $\left(25^{\circ} \mathrm{C}\right)$ under continuous fluorescent light. Fungal single-spore cultures were obtained from the different Fusarium colonies recovered.

\subsection{Analysis of Soil Samples}

Analysis consisted of drying the soil under aseptic conditions at room temperature $\left(20-25^{\circ} \mathrm{C}\right)$, crushing and sifting the samples through a $200 \mu \mathrm{m}$ sieve and adding $0.02 \mathrm{~g}$ of the sifted soil to a Fusarium-selective medium as described by Komada [27] modified by Tello et al. [28] (this modification contains, per L, $10 \mathrm{~g}$ galactose, $10 \mathrm{~g}$ agar, $1.25 \mathrm{~g}$ Pentachloronitrobenzene (PCNB) and $0.5 \mathrm{~g}$ streptomycine sulfate). Sixteen petri dishes per sample were used and divided into four blocks of four dishes. Plates were incubated at $25^{\circ} \mathrm{C}$ under continuous fluorescent light for 10 days. The mean number of colony forming units (CFU) per petri dish and the standard deviation of the mean were calculated for all Fusarium species and used as the basis for comparisons. 


\subsection{Morphological Characterization of Fungal Isolates}

Morphological approach was used to assign isolates to the Fusarium spp. level. Procedures and taxonomic criteria of Nelson et al. [29] and Leslie and Summerell [30] were followed.

\subsection{Molecular Characterization of Fungal Isolates}

Molecular identification of representative single-spore soil and plant isolates was confirmed by sequencing of a portion of the EF-1 $\alpha$ gene using primers ef1 and ef2 [31]. A multilocus sequence typing was carried out for 70 selected plant isolates: 31 F. oxysporum, 23 F. proliferatum and 16 F. redolens (Table 2). To do that, DNA was extracted from single-spore Fusarium isolates using the Isolate II Plant DNA Kit (Bioline, Toronto, Canada) following the manufacturer's instructions. The DNA concentration was determined on an ND-1000 NanoDrop spectrophotometer (Nano-Drop Products, Wilmington, DE, USA). PCR reactions were performed to amplify and partially sequence EF-1 $\alpha$ RPB1 and RPB2 genes from each selected isolate. PCR reactions contained 10× PCR Buffer (Intron Biotechnology, Inc., Seongnam, Gyeonggi, Korea), $2 \mathrm{mM} \mathrm{MgCl2,} 0.25 \mathrm{mM}$ each dNTP, $0.4 \mu \mathrm{M}$ each primer (Table S1), $1 \mathrm{U}$ of i-Pfu DNA polymerase (Intron Biotechnology, Inc., Seongnam, Gyeonggi, Korea) and 20-100 ng of genomic DNA. Amplifications were carried out at $95^{\circ} \mathrm{C}$ for $3 \mathrm{~min}$ followed by 35 cycles of $30 \mathrm{~s}$ at $95^{\circ} \mathrm{C}$, $20 \mathrm{~s}$ at 59 (for $E F-1 \alpha$ ), 57 (for RPB1) or $55^{\circ} \mathrm{C}$ (for $R P B 2$ ) and $60 \mathrm{~s}$ at $72{ }^{\circ} \mathrm{C}$. The size of the amplicons was resolved in 1.5\% agarose gels in 0.5× TAE (Tris-acetate-EDTA) buffer; then, they were stained with RedSafe nucleic acid staining solution (Intron Biotechnology, Inc., Seongnam, Gyeonggi, Korea) and visualized over an ultraviolet transilluminator. Amplicons were purified using the FavorPrep Gel/PCR purification kit (Favorgen, Ping-Tung, Taiwan) following the manufacturer's instructions and sequenced by STAB VIDA DNA Sequencing Service. Sequences were deposited in GenBank and compared with available sequences in GenBank and Fusarium-MLST databases by BLAST analysis. The whole fungal collection was maintained on potato dextrose agar and Komada's media and stored at $4{ }^{\circ} \mathrm{C}$ in the fungus collection of the IFAPA and in the Polytechnic University of Madrid. Representative isolates were maintained in $20 \%(v / v)$ glycerol at $-80^{\circ} \mathrm{C}$.

Table 2. The Fusarium plant isolates used in this study for phylogenetic analysis and GeneBank accessions.

\begin{tabular}{|c|c|c|c|c|c|c|c|}
\hline \multirow{2}{*}{ Origin } & \multirow{2}{*}{ Species } & \multirow{2}{*}{ Code } & \multirow{2}{*}{ Isolate Source } & \multirow{2}{*}{ Location } & \multicolumn{3}{|c|}{ Genbank Accession No. } \\
\hline & & & & & $E F-1 \alpha$ & $R P B 1$ & $R P B 2$ \\
\hline This Study & F. oxysporum & GR_FOAc & Asparagus Plant & Loja & МТ305183 & МТ305069 & MT305125 \\
\hline This study & F. oxysporum & GR_FOA5 & Asparagus plant & Loja & МТ305184 & MT305070 & MT305126 \\
\hline This study & F. oxysporum & GR_FOA8 & Asparagus plant & Loja & МТ305185 & MT305071 & MT305127 \\
\hline This study & F. oxysporum & GR_FOA13 & Asparagus plant & Loja & МТ305186 & MT305072 & MT305128 \\
\hline This study & F. oxysporum & GR_FOA14 & Asparagus plant & Loja & МТ305187 & МТ305073 & MT305129 \\
\hline This study & F. oxysporum & GR_FOA21 & Asparagus plant & Loja & МТ305188 & МТ305074 & MT305130 \\
\hline This study & F. oxysporum & GR_FOA125 & Asparagus plant & Loja & МТ305189 & MT305075 & MT305131 \\
\hline This study & F. oxysporum & GR_FOA128 & Asparagus plant & Loja & МТ305190 & МТ305076 & MT305132 \\
\hline This study & F. oxysporum & GR_FOA141 & Asparagus plant & Loja & МТ305191 & МТ305077 & МТ305133 \\
\hline This study & F. oxysporum & GR_FOA164 & Asparagus plant & Loja & MT305192 & МT305078 & МT305134 \\
\hline This study & F. oxysporum & GR_FOA168 & Asparagus plant & Loja & MT305193 & MT305079 & MT305135 \\
\hline This study & F. oxysporum & GR_FOA179 & Asparagus plant & Loja & MT305194 & MT305080 & MT305136 \\
\hline This study & F. oxysporum & GR_FOA230 & Asparagus plant & El Jau & МT305195 & MT305081 & МT305137 \\
\hline This study & F. oxysporum & GR_FOA247 & Asparagus plant & El Jau & МТ305196 & MT305082 & МT305138 \\
\hline This study & F. oxysporum & GR_FOA249 & Asparagus plant & El Jau & МТ305197 & MT305083 & МТ305139 \\
\hline This study & F. oxysporum & NA_FOA01 & Asparagus plant & Ancín & MT568933 & MT568949 & MT568965 \\
\hline This study & F. oxysporum & NA_FOA04 & Asparagus plant & Ancín & MT568934 & MT568950 & MT568966 \\
\hline This study & F. oxysporum & NA_FOA08 & Asparagus plant & Ancín & MT568935 & MT568951 & MT568967 \\
\hline This study & F. oxysporum & NA_FOA10 & Asparagus plant & Ancín & MT568936 & MT568952 & MT568968 \\
\hline This study & F. oxysporum & NA_FOA12 & Asparagus plant & Berbinzana & MT568937 & MT568953 & MT568969 \\
\hline This study & F. oxysporum & NA_FOA13 & Asparagus plant & Berbinzana & MT568938 & MT568954 & MT568970 \\
\hline This study & F. oxysporum & NA_FOA14 & Asparagus plant & Berbinzana & MT568939 & MT568955 & MT568971 \\
\hline This study & F. oxysporum & NA_FOA15 & Asparagus plant & Tudela & MT568940 & MT568956 & MT568972 \\
\hline This study & F. oxysporum & MA_FOA16 & Asparagus plant & Aranjuez & MT568941 & MT568957 & MT568973 \\
\hline This study & F. oxysporum & MA_FOA17 & Asparagus plant & Aranjuez & MT568942 & MT568958 & MT568974 \\
\hline
\end{tabular}


Table 2. Cont.

\begin{tabular}{|c|c|c|c|c|c|c|c|}
\hline \multirow{2}{*}{ Origin } & \multirow{2}{*}{ Species } & \multirow{2}{*}{ Code } & \multirow{2}{*}{ Isolate Source } & \multirow{2}{*}{ Location } & \multicolumn{3}{|c|}{ Genbank Accession No. } \\
\hline & & & & & $E F-1 \alpha$ & RPB1 & $R P B 2$ \\
\hline This study & F. oxysporum & MA_FOA19 & Asparagus plant & Aranjuez & MT568943 & MT568959 & MT568975 \\
\hline This study & F. oxysporum & MA_FOA20 & Asparagus plant & Aranjuez & MT568944 & MT568960 & MT568976 \\
\hline This study & F. oxysporum & MA_FOA23 & Asparagus plant & Aranjuez & MT568945 & MT568961 & MT568977 \\
\hline This study & F. oxysporum & MA_FOA24 & Asparagus plant & Aranjuez & MT568946 & MT568962 & MT568978 \\
\hline This study & F. oxysporum & MA_FOA25 & Asparagus plant & Aranjuez & MT568947 & MT568963 & MT568979 \\
\hline This study & F. oxysporum & MA_FOA27 & Asparagus plant & Aranjuez & MT568948 & MT568964 & MT568980 \\
\hline This study & F. proliferatum & GR_FPb & Asparagus plant & Loja & МТ305198 & МT305084 & МТ305140 \\
\hline This study & F. proliferatum & GR_FP3 & Asparagus plant & Loja & МТ305199 & МT305085 & МT305141 \\
\hline This study & F. proliferatum & GR_FP17 & Asparagus plant & Loja & МТ305201 & МТ305086 & МТ305143 \\
\hline This study & F. proliferatum & GR_FP24 & Asparagus plant & Loja & МТ305202 & МТ305087 & МТ305144 \\
\hline This study & F. proliferatum & GR_FP86 & Asparagus plant & Loja & МТ305203 & МТ305088 & МТ305145 \\
\hline This study & F. proliferatum & GR_FP87 & Asparagus plant & Loja & МT305204 & МТ305089 & МТ305146 \\
\hline This study & F. proliferatum & GR_FP100 & Asparagus plant & Loja & МТ305205 & МТ305090 & МТ305147 \\
\hline This study & F. proliferatum & GR_FP120 & Asparagus plant & Loja & МТ305206 & МТ305091 & МТ305148 \\
\hline This study & F. proliferatum & GR_FP130 & Asparagus plant & Loja & МТ305207 & МТ305092 & МТ305149 \\
\hline This study & F. proliferatum & GR_FP172 & Asparagus plant & Loja & МТ305208 & МТ305093 & МТ305150 \\
\hline This study & F. proliferatum & GR_FP182 & Asparagus plant & Loja & МТ305210 & МТ305094 & МТ305152 \\
\hline This study & F. proliferatum & GR_FP234 & Asparagus plant & El Jau & МТ305211 & МТ305095 & МТ305153 \\
\hline This study & F. proliferatum & GR_FP248 & Asparagus plant & El Jau & МТ305212 & МТ305096 & МТ305154 \\
\hline This study & F. proliferatum & NA_FPA01 & Asparagus plant & Larraga & MW091265 & MW091281 & MW091299 \\
\hline This study & F. proliferatum & NA_FPA02 & Asparagus plant & Ancín & MW091266 & MW091282 & MW091300 \\
\hline This study & F. proliferatum & MA_FPA04 & Asparagus plant & Aranjuez & MW091267 & MW091284 & MW091302 \\
\hline This study & F. proliferatum & MA_FPA06 & Asparagus plant & Aranjuez & MW091268 & MW091286 & MW091304 \\
\hline This study & F. proliferatum & MA_FPA08 & Asparagus plant & Aranjuez & MW091269 & MW091288 & MW091306 \\
\hline This study & F. proliferatum & MA_FPA10 & Asparagus plant & Aranjuez & MW091270 & MW091290 & MW091308 \\
\hline This study & F. proliferatum & MA_FPA11 & Asparagus plant & Aranjuez & MW091271 & MW091291 & MW091309 \\
\hline This study & F. proliferatum & MA_FPA12 & Asparagus plant & Aranjuez & MW091272 & MW091292 & MW091310 \\
\hline This study & F. proliferatum & MA_FPA15 & Asparagus plant & Aranjuez & MW091275 & MW091293 & MW091313 \\
\hline This study & F. proliferatum & MA_FPA16 & Asparagus plant & Aranjuez & MW091276 & MW091294 & MW091314 \\
\hline This study & F. redolens & GR_FRa & Asparagus plant & Loja & МТ305213 & МТ305097 & МТ305155 \\
\hline This study & F. redolens & GR_FR4 & Asparagus plant & Loja & МТ305214 & МТ305098 & МТ305156 \\
\hline This study & F. redolens & GR_FR7 & Asparagus plant & Loja & МТ305215 & МТ305099 & МТ305157 \\
\hline This study & F. redolens & GR_FR18 & Asparagus plant & Loja & МТ305216 & МT305100 & MT305158 \\
\hline This study & F. redolens & GR_FR20 & Asparagus plant & Loja & МТ305217 & МT305101 & МТ305159 \\
\hline This study & F. redolens & GR_FR23 & Asparagus plant & Loja & МТ305218 & МТ305102 & МТ305160 \\
\hline This study & F. redolens & GR_FR29 & Asparagus plant & Loja & МТ305219 & МТ305103 & МТ305161 \\
\hline This study & F. redolens & GR_FR58 & Asparagus plant & Loja & МТ305220 & МТ305104 & МТ305162 \\
\hline This study & F. redolens & GR_FR104 & Asparagus plant & Loja & МТ305221 & МТ305105 & МТ305163 \\
\hline This study & F. redolens & GR_FR157 & Asparagus plant & Loja & МТ305224 & МТ305107 & МТ305165 \\
\hline This study & F. redolens & GR_FR186 & Asparagus plant & Loja & МТ305225 & МТ305108 & МТ305166 \\
\hline This study & F. redolens & GR_FR215 & Asparagus plant & El Jau & МТ305226 & МТ305109 & МТ305167 \\
\hline This study & F. redolens & NA_FRA01 & Asparagus plant & Ancín & MW091277 & MW091295 & MW091315 \\
\hline This study & F. redolens & NA_FRA02 & Asparagus plant & Ancín & MW091278 & MW091296 & MW091316 \\
\hline This study & F. redolens & NA_FRA03 & Asparagus plant & Ancín & MW091279 & MW091297 & MW091317 \\
\hline This study & F. redolens & NA_FRA04 & Asparagus plant & Berbinzana & MW091280 & MW091298 & MW091318 \\
\hline GenBank & F. oxysporum & RBG5769 & Soil & Australia & KJ397041 & KJ397185 & KJ397221 \\
\hline GenBank & F. oxysporum & RBG5796 & Soil & Australia & KJ397061 & KJ397205 & KJ397241 \\
\hline GenBank & F. oxysporum & RBG5801 & Soil & Australia & KJ397062 & KJ397206 & KJ397242 \\
\hline GenBank & F. oxysporum & RBG5807 & Soil & Australia & KJ397066 & KJ397210 & KJ397246 \\
\hline GenBank & F. oxysporum & RBG5765 & Soil & Australia & KJ397075 & KJ397219 & KJ397255 \\
\hline GenBank & F. oxysporum & RBG5771 & Soil & Australia & KJ397042 & KJ397186 & KJ397222 \\
\hline GenBank & F. oxysporum & RBG5782 & Soil & Australia & KJ397051 & KJ397195 & KJ397231 \\
\hline GenBank & F. oxysporum & RBG5818 & Soil & Australia & KJ397072 & KJ397216 & KJ397252 \\
\hline GenBank & F. oxysporum & RBG5768 & Soil & Australia & KJ397040 & KJ397184 & KJ397220 \\
\hline GenBank & F. oxysporum & RBG5779 & Soil & Australia & KJ397048 & KJ397192 & KJ397228 \\
\hline GenBank & F. oxysporum & RBG5781 & Soil & Australia & KJ397050 & KJ397194 & KJ397230 \\
\hline GenBank & F. oxysporum & RBG5784 & Soil & Australia & KJ397053 & KJ397197 & KJ397233 \\
\hline GenBank & F. oxysporum & RBG5697 & Soil & Australia & KJ397064 & KJ397208 & KJ397244 \\
\hline GenBank & F. oxysporum & RBG5783 & Soil & Australia & KJ397052 & KJ397196 & KJ397232 \\
\hline GenBank & F. oxysporum & RBG5794 & Soil & Australia & KJ397060 & KJ397204 & KJ397240 \\
\hline GenBank & F. oxysporum & RBG5817 & Soil & Australia & KJ397071 & KJ397215 & KJ397251 \\
\hline
\end{tabular}


Table 2. Cont.

\begin{tabular}{|c|c|c|c|c|c|c|c|}
\hline \multirow{2}{*}{ Origin } & \multirow{2}{*}{ Species } & \multirow{2}{*}{ Code } & \multirow{2}{*}{ Isolate Source } & \multirow{2}{*}{ Location } & \multicolumn{3}{|c|}{ Genbank Accession No. } \\
\hline & & & & & $E F-1 \alpha$ & RPB1 & RPB2 \\
\hline GenBank & F. commune & $\begin{array}{l}\text { NRRL } \\
28387\end{array}$ & $\begin{array}{l}\text { Dianthus } \\
\text { caryophyllus }\end{array}$ & Netherlands & HМ057338 & JX171525 & JX171638 \\
\hline GenBank & F. proliferatum & $\begin{array}{l}\text { NRRL } \\
62905\end{array}$ & Zea mays & USA & MN193865 & MN193921 & MN193893 \\
\hline GenBank & F. proliferatum & $\begin{array}{l}\text { NRRL } \\
43617\end{array}$ & Human & USA & HM347124 & HM347185 & EF470206 \\
\hline GenBank & F. fujikuroi & $\begin{array}{l}\text { NRRL } \\
5538\end{array}$ & $\begin{array}{l}\text { Saccharum } \\
\text { officinarum }\end{array}$ & Taiwan & MN193860 & MN193916 & MN193888 \\
\hline GenBank & F. concentricum & $\begin{array}{l}\text { NRRL } \\
25181\end{array}$ & Musa sapientum & Costa Rica & MT010992 & MT010942 & MT010981 \\
\hline GenBank & F. sacchari & $\begin{array}{l}\text { NRRL } \\
66326\end{array}$ & Lab cross & USA & MN193868 & MN193924 & MN193896 \\
\hline GenBank & F. bulbicola & $\begin{array}{l}\text { NRRL } \\
13618\end{array}$ & Nerine bowdenii & Germany & KF466415 & KF466394 & KF466404 \\
\hline GenBank & F. subglutinans & $\begin{array}{l}\text { NRRL } \\
22016\end{array}$ & Zea mays & USA & HМ057336 & JX171486 & JX171599 \\
\hline GenBank & F. begoniae & $\begin{array}{l}\text { NRRL } \\
25300\end{array}$ & Begonia elatior & Germany & MN193858 & MN193914 & MN193886 \\
\hline GenBank & F. verticillioides & $\begin{array}{l}\text { NRRL } \\
20956\end{array}$ & Zea mays & USA & MN193873 & MN193929 & MN193901 \\
\hline GenBank & F. nygamai & $\begin{array}{l}\text { NRRL } \\
26421\end{array}$ & Human & Egypt & HM347121 & HM347147 & EF470127 \\
\hline GenBank & $F$, napiforme & $\begin{array}{l}\text { NRRL } \\
25196\end{array}$ & $\begin{array}{l}\text { Pennisetum } \\
\text { typhoides }\end{array}$ & $\begin{array}{l}\text { South } \\
\text { Africa }\end{array}$ & MN193863 & MN193919 & MN193891 \\
\hline GenBank & F. oxysporum & $\begin{array}{l}\text { NRRL } \\
26360\end{array}$ & Human & USA & HM347120 & НМ347146 & EF470126 \\
\hline GenBank & F. redolens & $\begin{array}{l}\text { NRRL } \\
54967\end{array}$ & Feline & USA & KC808221 & KC808300 & KC808363 \\
\hline GenBank & F. redolens & $\begin{array}{l}\text { NRRL } \\
22901\end{array}$ & $\begin{array}{l}\text { Pseudotsuga } \\
\text { menziesii }\end{array}$ & Canada & MT409452 & MT409432 & JX171616 \\
\hline GenBank & F. spartum & $\begin{array}{l}\text { NRRL } \\
66894\end{array}$ & $\begin{array}{l}\text { Macrochloa } \\
\text { tenacissima }\end{array}$ & Tunisia & MT409457 & MT409437 & MT409447 \\
\hline GenBank & F. hostae & $\begin{array}{l}\text { NRRL } \\
29888\end{array}$ & Hosta sp. & USA & MT409455 & MT409435 & MT409445 \\
\hline
\end{tabular}

\subsection{Pathogenicity Tests of Plant and Soil Isolates}

In total, 37 Fusarium monoconidial isolates (F. oxysporum $(n=10)$, F. redolens $(n=8)$, F. proliferatum $(n=11)$ and Fusarium solani species complex (FSSC) $(n=8))$ from diseased plants were tested for pathogenicity. For this purpose, asparagus seeds of cultivar "Grande F1" were surface disinfested by immersion with $3.5 \%$ sodium hypochlorite for $20 \mathrm{~min}$ and subsequently rinsed with tap water and incubated in sterile wet paper at $28^{\circ} \mathrm{C}$ in the dark. Fungal isolates were cultured in Czapek-Dox broth on a rotary shaker $(150 \mathrm{rpm})$ at $25^{\circ} \mathrm{C}$ for 10 days. Once plants reached an appropriate size, they were inoculated by immersion of the roots in a suspension of $10^{6}-10^{7} \mathrm{CFU} / \mathrm{mL}$ for $30 \mathrm{~min}$. Non-inoculated control plants were root-dipped in sterile Czapek-Dox broth. The inoculated plants were transplanted into pots with $1.6 \mathrm{~L}$ of sterile vermiculite. Three plants were sown per pot and 12 plants for each Fusarium isolate. Plants were maintained in a growth chamber set at $25 / 18^{\circ} \mathrm{C}$ (light/dark) with a $14 \mathrm{~h}$ photoperiod (14,000 lux) for 60 days. These experiments were carried out twice for each Fusarium species tested.

On the other hand, 258 Fusarium isolates (F. oxysporum (sensu Leslie \& Summerel, 2006) $(n=87)$, FSSC $(n=68)$, F. equiseti $(n=41)$, F. acuminatum $(n=32)$, F. compactum $(n=16)$ and F. proliferatum $(n=14))$ from soil samples were tested for pathogenicity in a first set of pot experiments screening. This set of tests consisted of four sequential experiments. All isolates were inoculated by drenching the potting substrate (twice autoclaved vermiculite, $1 \mathrm{~h}$ at $120^{\circ} \mathrm{C}$ each) contained in $200-\mathrm{mL}$ plastic pots with $80 \mathrm{~mL}$ inoculum. The inocula consisted of $10^{4}-10^{5} \mathrm{CFU} / \mathrm{mL}$ suspensions of each isolate in sterile water. The inoculum was prepared by grinding colonies fully covering the entire surface of PDA 
(F. oxysporum, FSSC and F. proliferatum isolates) or KCL-agar (F. equiseti, F. compactum and F. acuminatum) plates. Isolates grew at $25^{\circ} \mathrm{C}$ for 10-12 days in darkness for PDA plates and for 14-15 days under UV light for $\mathrm{KCl}$ plates. Non-inoculated control plants were watered with an aqueous homogenize of non-colonized PDA. Seeds were disinfected as described above. Inoculations occurred when asparagus plants cv. Grande F1 had emerged on the substrate. Three plants were sown per pot and six plants were inoculated per isolate. Pots were randomly distributed and maintained for 30 days in a growth chamber under the same conditions explained above. The incidence of plants showing symptoms of infection of the roots was assessed at the end of the experiment to determine which isolates expressed pathogenicity (infection of the roots clearly higher than the control plants) for a further evaluation through a second set of inoculations.

The next set of experiments was conducted sequentially to evaluate 61 soil isolates, previously demonstrated pathogenic: F. oxysporum $(n=49)$ and F. proliferatum (12). Experiments were performed as described above for the tests of plant isolates but using 1-L plastic pots with three replicates containing seven plants each.

Plants were evaluated weekly for disease severity based on a $1-5$ scale, where $1=1-20 \%$, $2=21-40 \%, 3=41-60 \%, 4=61-80 \%$ and $5=81-100 \%$ of the fronds showing chlorosis, necrosis or wilt. Area under disease progress curves (AUDPC) was calculated for each species by clustering recorded data from all plant isolates [32]. At the end of each experiment, the plants were removed from the pots, the roots rinsed in tap water, and the severity of the lesions on the root system was assessed on the same scale as fronds, related to necrosis in the root system. The fronds and root wet weights were also measured. Mean \pm standard error of root rot severity and fronds severity ratings and weight loss with respect to the control, over 12 replicate plants inoculated with each isolate, were determined. The proportion of plants that were dead was also assessed at the end of the experiments. All the dead plants and several randomly assigned symptomatic plants per isolate were analyzed for re-isolation on PDA.

\subsection{Phylogenetic Analysis}

Phylogenetic analyses of the 70 plant isolates of F. oxysporum, F. proliferatum and F. redolens species were carried out by the analysis of three phylogenetically informative loci: the translation elongation factor- $1 \alpha(E F-1 \alpha)$ and the DNA-directed RNA polymerase II largest (RPB1) and second largest subunits (RPB2). These loci were chosen due to be the most informative for species-level identifications and to be well represented in the database [33-36]. These sequence fragments were manually concatenated (2 598 nucleotides). In addition, sequences from Fusarium oxysporum species complex (FOSC), Fusarium fujikuroi species complex (FFSC) and Fusarium redolens species complex (FRSC) retrieved from GenBank were included in the analyses. For the analysis of FOSC, some GenBank isolates were used belonging to Clades 1-4 defined by $\mathrm{O}^{\prime}$ Donnell et al. [37] and later grouped into PS1 and PS2 phylogenetic species according to Laureance et al. [38]. Multiple sequence alignments were performed in MEGA7 software using the CLUSTALW algorithm refined with MUSCLE and edited manually. Phylogenetic analyses were conducted using MEGA7 through maximum likelihood (ML) analysis using the kimura-2 parameter model for the analysis of FOSC, FFSC and FRSC isolates independently, all of them with a rate of variation across sites [39]. Support for internal branches was assessed by 1000 ML bootstrapped pseudo replicates of data. Nodes with bootstrap support $\geq 70 \%$ were indicated in the final trees.

\subsection{Genetic Diversity}

Haplotype distribution of the asparagus isolates belonging to FOSC, FFSC and FRSC was performed using DNA sequence information from concatenated loci by DnaSP v5 software [40]. Number of haplotypes (h), haplotype (gene) diversity $(\mathrm{H})$ and nucleotide diversity $(\pi)$ of the species complexes were calculated by DnaSP v5. Fusarium oxysporum plant isolates were artificially grouped into three populations corresponding to the three prospected production areas to discern potential 
differences among populations. Chi square statistic [41] was used to calculate genetic differentiation among populations.

\subsection{Statistical Analysis of Data}

Analysis of variance on the Fusarium isolates from soil at different crop ages (0-22 years), average temperature of the warmest month, altitude (meters over the sea level) and type of consumption (white asparagus vs. green asparagus), as well as for Fusarium spp. isolated from feeder and storage roots and those used for pathogenicity test evaluation (fronds and root weights), were performed using Fisher's least significant difference (LSD) tests at 99.9\% confidence were carried out using STATGRAPHICS Centurion XVIII statistical package software (StatPoint, Inc., Herndon, VA, USA). Additional non-parametric Kolmogorov-Smirnov, Mann-Whitney-Wilcoxon and Kruskal-Wallis tests were performed when the assumption of the normality of the distributions for the two samples studied was not sustainable (disease severity on fronds). Differences among pathogenicity trials were not found $(p \geq 0.05)$, thus data were clustered for consistency of analysis. Arcsine square root transformation was applied to weight loss percentages.

To determine direct correlation between the density of inoculum of any of the Fusarium species and the Field Disease Index (after arcsine square root transformation), simple regression analysis was adjusted to the non-linear Y-square model as it showed the highest $R^{2}$ value. The same statistical package software was used for the generalized linear model $\left(Y_{i}=\beta_{0}+\beta_{1} X_{1, i}+\beta_{2} X_{2, i}+\beta_{3} X_{3, i}+\ldots+\right.$ $\beta_{\mathrm{k}} X_{\mathrm{k}, \mathrm{i}}+\varepsilon_{\mathrm{i}}$ ), where " $\mathrm{Y}$ " is the response variable (Field Disease Index) " $\mathrm{B}_{1} \mathrm{X}_{\mathrm{k}, \mathrm{i}}$ " the predictor variables and " $\mathrm{i}$ " the error. To determine when a pair of variables was effectively correlated, the $p$-value of its correlation coefficient was calculated (correlation coefficient test). If the $p$-value is less than or equal to 0.05 , the linear correlation of the two variables is statistically significant at $5 \%$ confidence level.

\section{Results}

\subsection{Characterization of Fusarium Soil Communities and Correlation with Climatic Features}

Eight species belonging to seven species complexes of the genus Fusarium were identified from the analyzed soil samples: F. oxysporum from FOSC (F. oxysporum species complex), F. proliferatum from FFSC (F. fujikuroi species complex), F. redolens from FRSC (F. redolens species complex), F. solani sensu stricto from FSSC ( $F$. solani species complex), F. equiseti from FIESC (F. incarnatum-equiseti species complex), F. culmorum and F. compactum from FSAMSC (F. sambicinum species complex) and F. acuminatum from FTSC (F. tricinctum species complex). All three provinces analyzed showed differences in the relative frequencies of isolation of each species, although the two areas with the longest history of cultivation, Navarra and Andalusia, showed similar patterns of distribution of the main species isolated (Figure 2).

The predominant species in the fields of both provinces was F. oxysporum, belonging to FOSC. This species presents the highest percentages of colonies per gram of soil analyzed (Figure 2), with average values of $2893 \pm 1935$ CFU/g in Navarra and $1126 \pm 933$ CFU/g in Andalusia. The second specific group in importance in both provinces is FIESC, followed by the isolates belonging to FSSC. The distribution in the region of Madrid varies considerably. The isolates belonging to the FIESC appear in a greater number in the soil samples of Madrid than in the other two regions, with mean values of $3240 \pm 1343 \mathrm{CFU} / \mathrm{g}$, much higher than the inoculum density of FSSC isolates and almost ten times higher than the values of the FOSC isolates.

F. proliferatum was isolated in similar amounts in the three sampled regions. F. redolens was isolated from soil samples of Navarra with an average of $287 \pm 364 \mathrm{CFU} / \mathrm{g}$ of soil and from soils of Andalusia (with a density of inoculum not determined). F. acuminatum and F. compactum were isolated only from Andalusia. F. culmorum was isolated from the northern and central zones. 

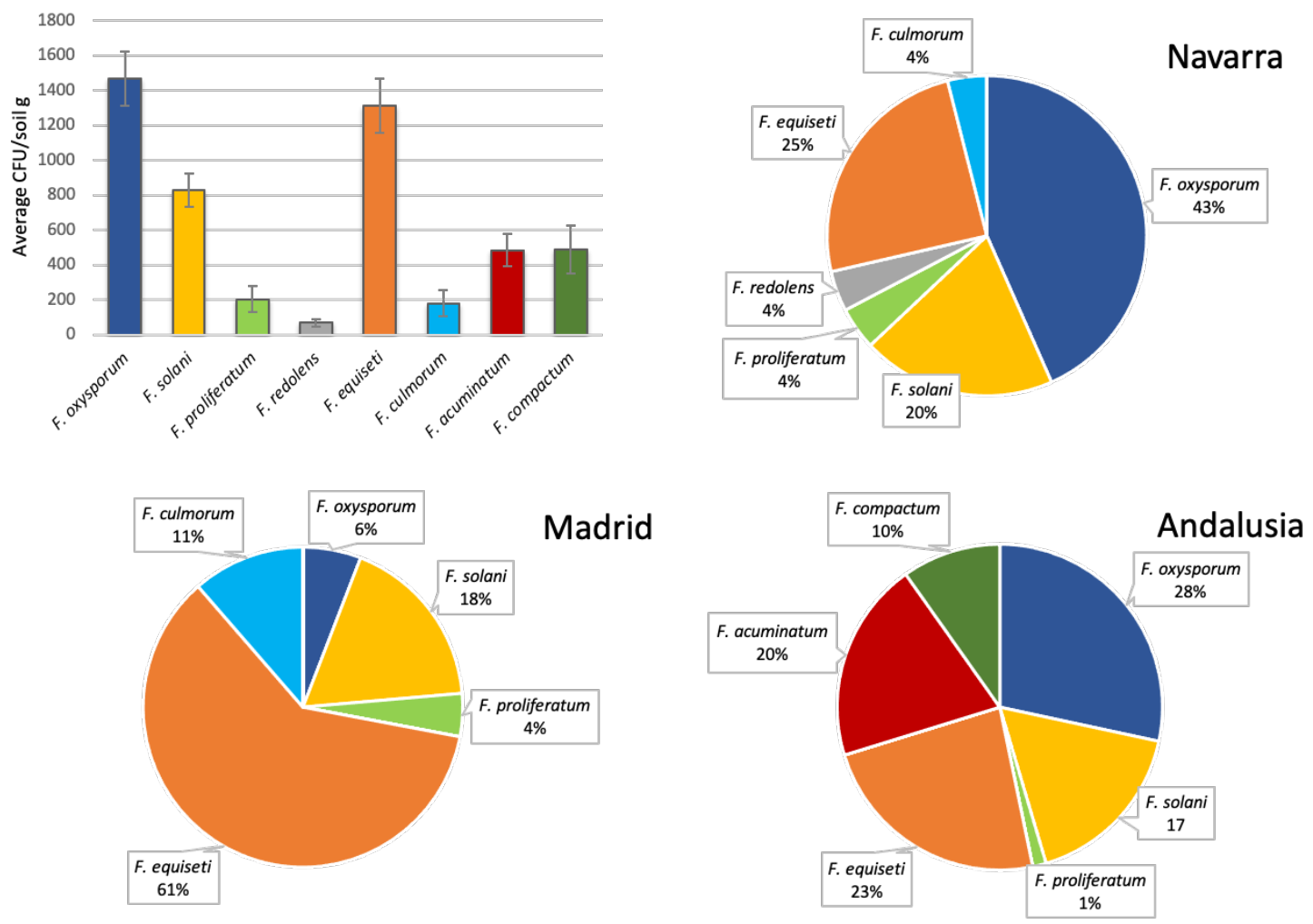

Figure 2. Average values of CFU/g of soil for the different Fusarium spp. and relative frequencies of isolation of Fusarium soil communities in the three analyzed production regions.

The age of the crop had no statistically significant effects on the total density of Fusarium inoculum (UFC/g of soil) ( $p=0.090)$. No direct correlation was detected between the density of inoculum of any of the species and the Field Disease Index (FDI) values observed during the initial sampling in Madrid and Navarra fields except for F. oxysporum. The simple regression analysis showed $p$-value $=0.034$ for F. oxysporum, so the linear correlation with FDI is statistically significant at $5 \%$ confidence level. F. oxysporum colonies showed the highest correlation with $\mathrm{R}^{2}$ value of $10.25 \%$ (Table 3 ).

Table 3. Equations and correlation coefficients between Field Disease Index (FDI) and Colony Forming Units (CFU) per gram of soil analyzed, evaluated for every Fusarium species.

\begin{tabular}{|c|c|c|c|c|c|}
\hline Adjustment & $n$ & Adjusted Equation & $\begin{array}{l}\text { Correlation } \\
\text { Coefficient }\end{array}$ & $p$-Value & $\mathbf{R}^{2}$ \\
\hline $\mathrm{Y}^{2}$ equation & 44 & $\begin{array}{c}\text { FDI }=\text { sqrt }(256.328-0.184087 \cdot \mathrm{CFU} \\
\text { F. oxysporum } / \mathrm{g} \text { soil })\end{array}$ & 0.320118 & 0.0341 & $10.25 \%$ \\
\hline $\mathrm{Y}^{2}$ equation & 44 & $\begin{array}{c}\text { FDI }=\operatorname{sqrt}(858.517-0.211922 \cdot \mathrm{CFU} \\
\text { F. solani/g soil })\end{array}$ & -0.131103 & 0.3963 & $1.72 \%$ \\
\hline $\mathrm{Y}^{2}$ equation & 44 & $\begin{array}{c}\mathrm{FDI}=\operatorname{sqrt}(639.741+0.0475964 \cdot \mathrm{CFU} \\
\text { F. proliferatum } / \mathrm{g} \text { soil })\end{array}$ & 0.0334676 & 0.8292 & $0.11 \%$ \\
\hline $\mathrm{Y}^{2}$ equation & 44 & $\begin{array}{c}\text { FDI }=\text { sqrt }(709.614-0.29721 \cdot \mathrm{CFU} \\
\text { F. redolens } / \mathrm{g} \text { soil })\end{array}$ & -0.0913385 & 0.5554 & $0.83 \%$ \\
\hline $\mathrm{Y}^{2}$ equation & 44 & $\begin{array}{c}\text { FDI }=\text { sqrt }(411.435+0.121763 \cdot \mathrm{CFU} \\
\text { F. incarnatum } / \mathrm{g} \text { soil })\end{array}$ & 0.170733 & 0.2678 & $2.91 \%$ \\
\hline $\mathrm{Y}^{2}$ equation & 44 & $\begin{array}{c}\mathrm{FDI}=\operatorname{sqrt}(571.778+0.19812 \cdot \mathrm{CFU} \\
\text { F. culmorum } / \mathrm{g} \text { soil }\end{array}$ & 0.192981 & 0.2094 & $3.72 \%$ \\
\hline
\end{tabular}


A high correlation $\left(\mathrm{R}^{2}=88 \%\right)$ was detected between F. oxysporum inoculum density and the average temperature of the warmest month of the year (Figure 3). The results indicate that the F. oxysporum inoculum increases significantly as temperature does, coinciding with recent works where the abundance of soil pathogens was directly linked to temperature [42] (Delgado-Baquerizo et al., 2020).

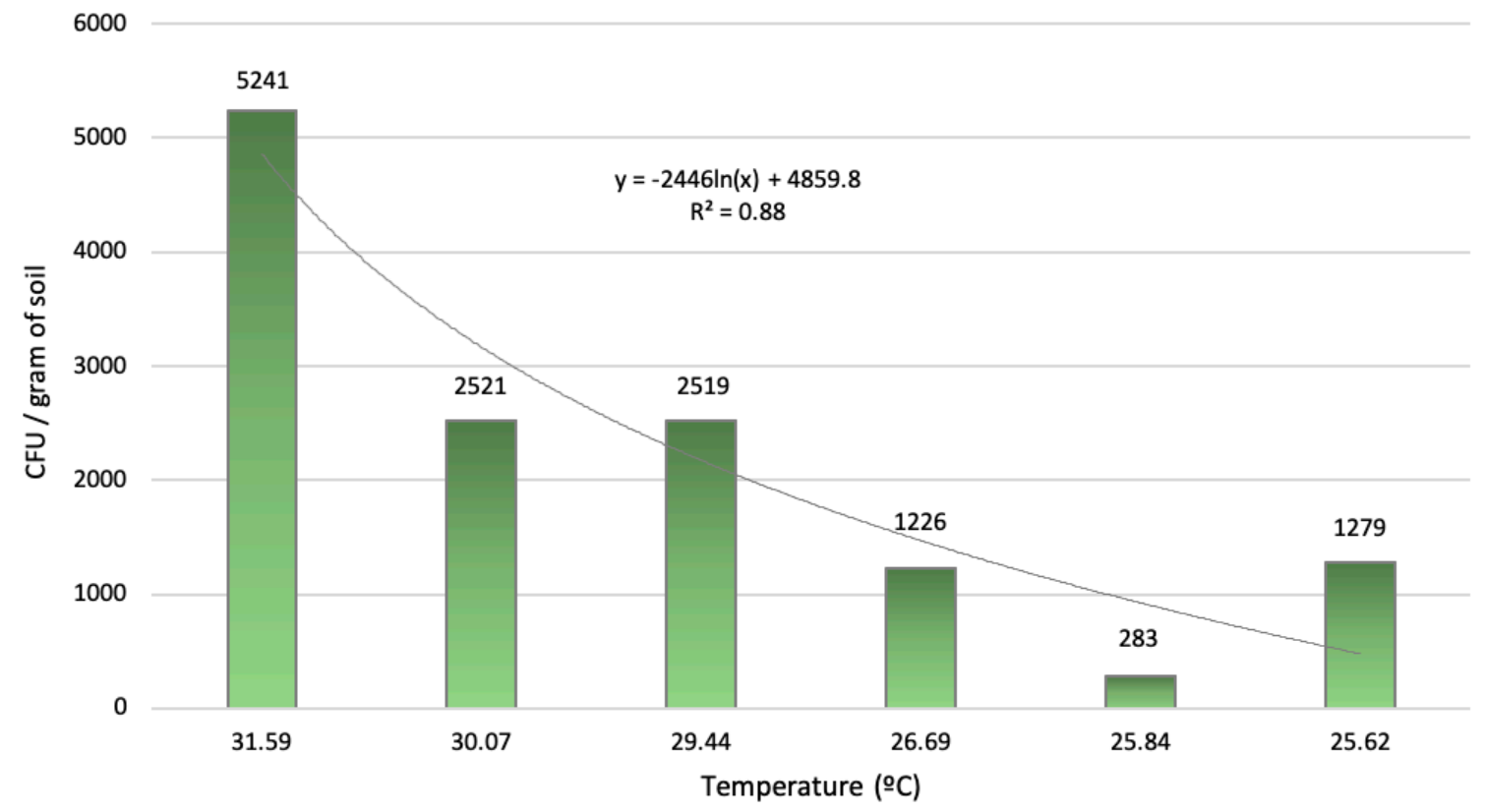

Figure 3. Fusarium oxysporum soil inoculum density isolated from sampled fields depending on the average temperature of the warmest month.

The GLM method allowed estimating the repeatability and reproducibility of the FDI measurement R-Squared $=59.27 \%$ (Adjusted R-Squared $=53.10 \%$ ). After the stepwise variable selection, five effects were selected in the model, including year of cultivation, inoculum density (or CFU/g of soil) and second-order interactions (Field Disease Index $=-4,6-(0.077917 \cdot \mathrm{CFU})+\left(0.585313 \cdot \mathrm{T}^{\mathrm{re}}\right)+(1.52425 \cdot$ Years $)$ $+\left(0.00245418 \cdot \mathrm{CFU} \cdot \mathrm{T}^{\mathrm{re}}\right)+(0.00130563 \cdot \mathrm{CFU} \cdot$ Years $)$.

The fact that this is a multiannual crop has made it possible to include the age of the crop in the model. The difficulty of modeling a disease caused by a soil fungus is evident, but contour plots have proved useful for establishing the response values. The 3D surface contour plot shown in Figure 4 allows understanding the relationship between the three main factors (average temperature, crop age and inoculum density) and the FDI response values. All three variables affect FDI values of affected fields. The dark blue region identifies the lowest percent FDI, and it decreases as the amount of inoculum in the soil increases. Temperature has an effect, but a smaller one: FDI increased rapidly within the temperature, although with moderate or low inoculum levels this effect does not translate into field damage. The contour levels reveal a peak of affected plants (\%) in fields with more than eight years and $6000 \mathrm{CFU} / \mathrm{g}$ of soil of $F$. oxysporum. FDI scores in this peak region are greater than $90 \%$. At that soil inoculum levels, intermediate FDI values can be observed at all temperature regimes in fields between three and seven years age (Figure 4). 


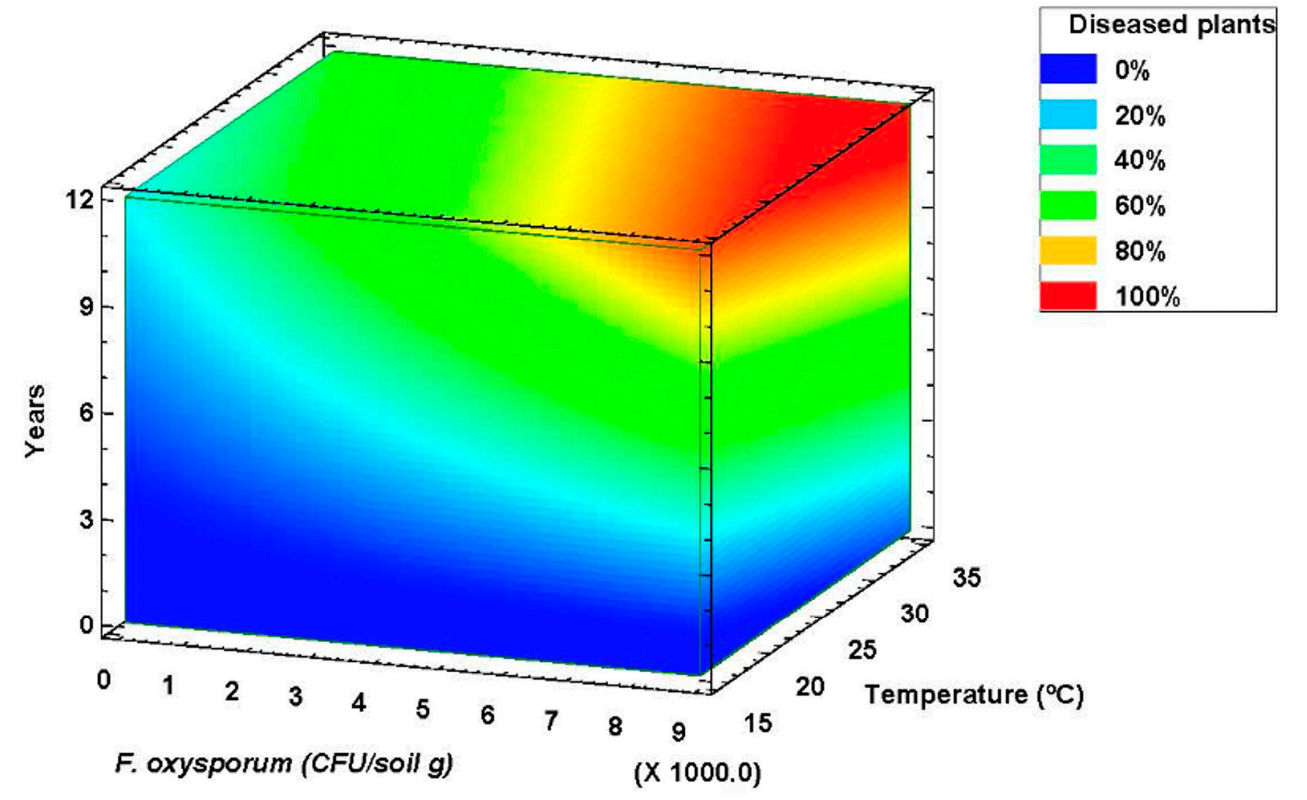

Figure 4. Disease Severity Index response surface contour plot at varying Fusarium oxysporum soil inoculum density, average temperature of the warmest month and number of years after asparagus plantation.

The number of F. oxysporum colonies per gram of soil isolated from fields dedicated to white and green asparagus was also analyzed. The comparative study allowed determining statistically significant differences $\left(p=2.57 \times 10^{-8}\right)$ between both asparagus types. The distributions of the CFU/g of soil of F. oxysporum/g are displayed in the boxplots below (Figure 5). The number of CFU/g soil isolated from green asparagus fields varies much less than that of white ones.

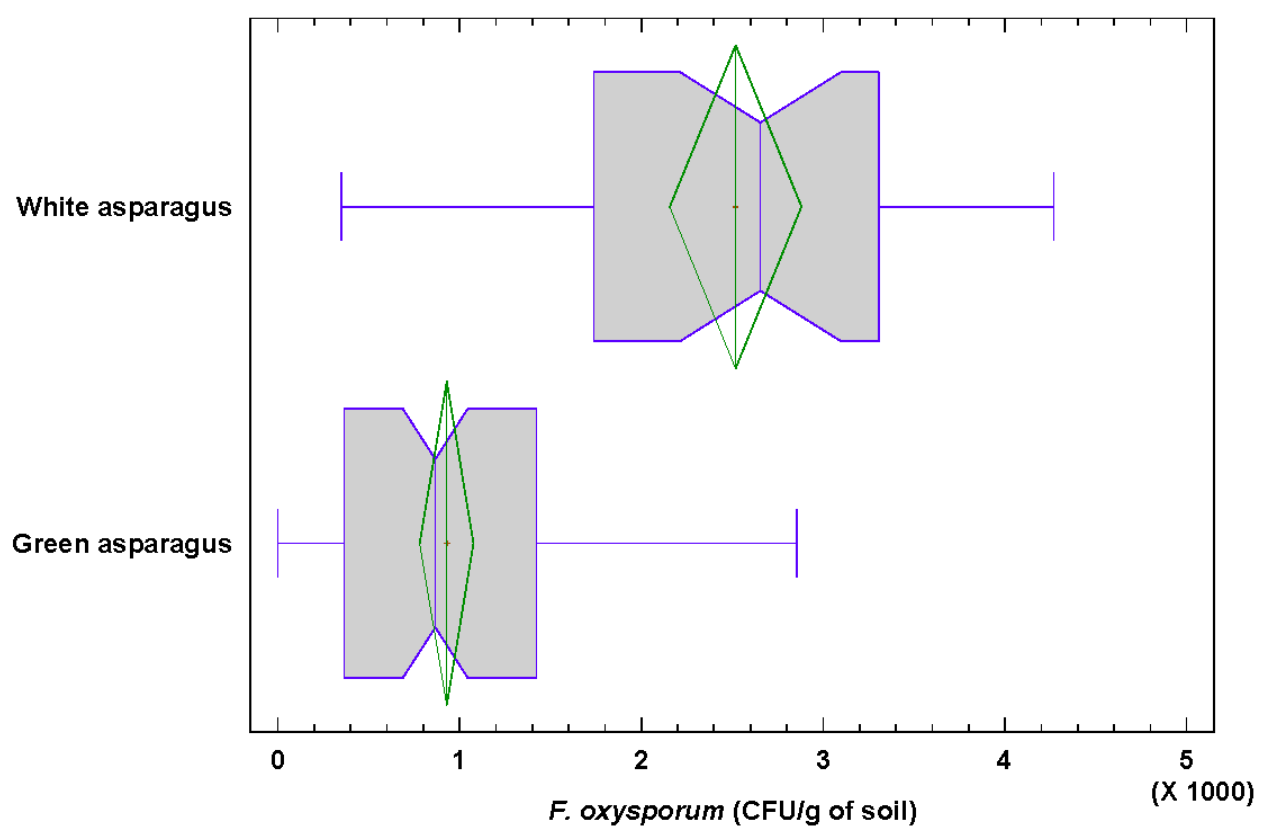

Figure 5. Boxplots showing mean CFU/g of soil depending on the cultivation type. Right of the box is the 75th percentile, the left is the 25 th percentile and the whiskers represent the maximum and minimum values. 
The average values of soil were $2860 \pm 1649 \mathrm{CFU} / \mathrm{g}$ for white asparagus fields and $1105 \pm 1082 \mathrm{CFU} / \mathrm{g}$ for green asparagus ones. The $25 \%(\mathrm{Q} 3)$ highest CFU/g of soil in white asparagus fields were all higher than the maximum level for green asparagus fields.

The maximum distance obtained in the Kolmogorov-Smirnov test, denoted by DN, is equal to 0.622 for the CFU/g of soil data. The $p$-value is less than $0.05\left(p=2.57 \times 10^{-8}\right)$, so there is a significant difference between the CFU distributions of $F$. oxysporum in white and green asparagus fields at $5 \%$ significance level.

\subsection{Characterization of Fusarium Plant Communities}

In total, 430 Fusarium isolates were obtained from 215 affected plants analyzed. Thirteen species were identified from the root system of the affected plants belonging to seven species complexes: F. oxysporum (FOSC); F. proliferatum and F. nygamai (FFSC); F. redolens (FRSC), F. solani sensu stricto, F. falciforme, F. tonkinense and F. eumartii (FSSC); F. culmorum, F. brachygibossum and F. graminearum (FSAMSC); F. avenaceum (FTSC); and F. equiseti (FIESC). F. oxysporum was the most prevalent species isolated from diseased plants roots, and the rest of Fusarium species detected showed low percentages of isolation (Figure 6). The isolation percentages of the different species vary according to the type of root (feeder or storage root), although only the isolation percentages of F. oxysporum showed statistically significant differences $(p=0.000)$ between the type of root analyzed. Isolation rate of $F$. oxysporum from feeder roots was $54.74 \%$, and $26.60 \%$ from storage roots.

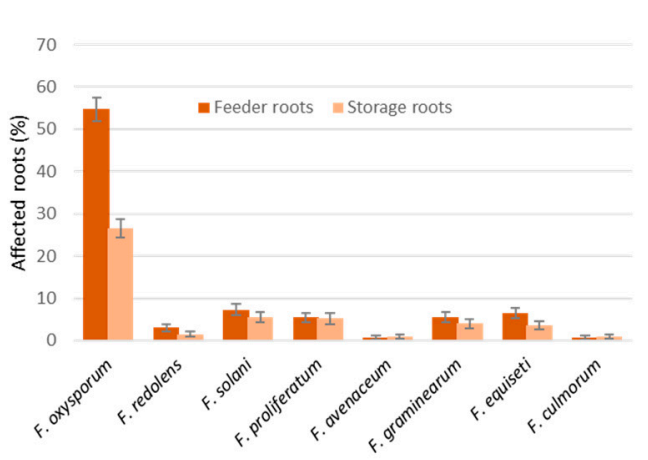

Madrid

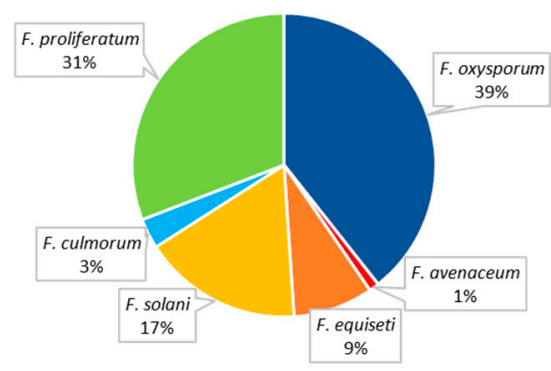

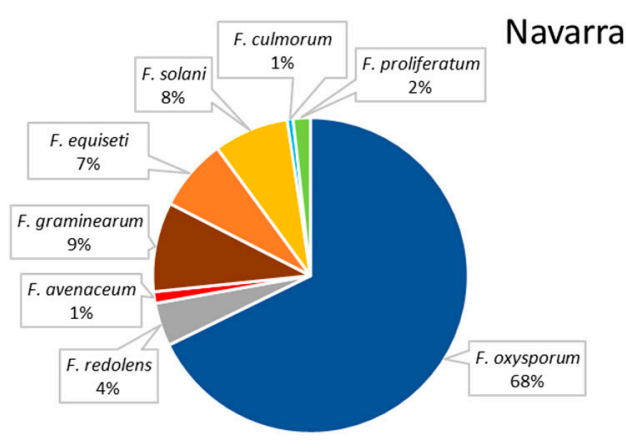

Andalusia

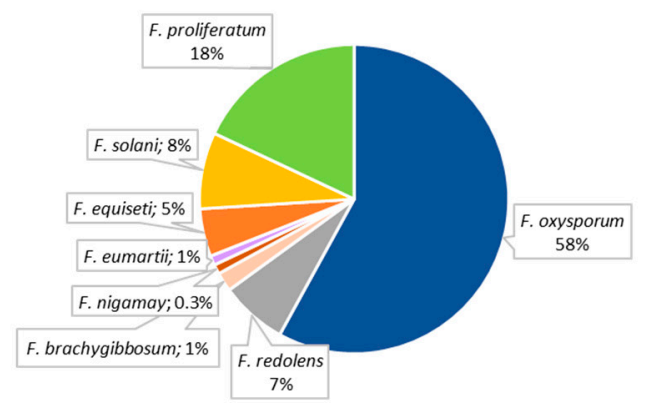

Figure 6. Average values of isolation from asparagus plants for the different Fusarium spp. depending on the root type and relative frequencies of isolation of Fusarium communities in the three analyzed production regions.

\subsection{Pathogenicity of Fusarium Species Associated to Asparagus}

The pathogenicity tests revealed that $90 \%$ of $F$. oxysporum, $87.5 \%$ of $F$. redolens and $90.9 \%$ of $F$. proliferatum plant isolates were pathogenic to asparagus, while a first screening revealed that $64.4 \%$ of the F. oxysporum and $92.9 \%$ of the F. proliferatum soil isolates were pathogenic to asparagus. None of the FSSC, F. equiseti, F. compactum and F. acuminatum isolates expressed any symptom on asparagus seedlings (Table 4). The isolates inoculated were re-isolated from inoculated plant tissues. 
Table 4. Fusarium spp. monoconidial isolates from symptomatic asparagus plants and soils of asparagus monoculture fields, tested for pathogenicity in pot experiments under growth-chamber conditions.

\begin{tabular}{ccccccc}
\hline & \multicolumn{5}{c}{ Fusarium Species } \\
\hline $\begin{array}{c}\text { Fusarium sp. } \\
\text { Plant Isolates }\end{array}$ & F. oxysporum & F. proliferatum & F. redolens & FSSC \\
\hline Number of isolates & 10 & 11 & 8 & 8 & & \\
Number of pathogenic & 9 & 10 & 7 & 0 & & \\
Percent pathogenic & 90 & 90.9 & 87.5 & 0 & & \\
\hline $\begin{array}{c}\text { Fusarium sp. } \\
\text { soil isolates }\end{array}$ & F. oxysporum & F. proliferatum & F. equiseti & FSSC & F. compactum & F. acuminatum \\
\hline Number of isolates & 87 & 14 & 41 & 68 & 16 & 0 \\
Number of pathogenic & 56 & 13 & 0 & 0 & 0 & 0 \\
Percent pathogenic & 64.4 & 92.9 & 0 & 0 & 0 & 0 \\
\hline
\end{tabular}

In general, F. proliferatum isolates produced the most severe symptoms, followed by F. oxysporum and $F$. redolens (Table 5). However, aggressiveness was not uniform among isolates belonging to the same species. The distribution of isolates by their aggressiveness based on plant mortality showed that F. proliferatum was the most aggressive species.

Table 5. Mean severity on roots and percentage of frond and root weight loss of "Grande F1" asparagus plants inoculated with Fusarium spp. isolates grown under growth-chamber conditions.

\begin{tabular}{|c|c|c|c|}
\hline $\begin{array}{l}\text { Fusarium sp. } \\
\text { Plant Isolates }\end{array}$ & Severity on Roots & Frond Weight Loss (\%) & Root Weight Loss (\%) \\
\hline F. proliferatum $(n=11)$ & $3.0 \pm 0.2 \mathrm{~A}$ & $59.0 \pm 3.9 \mathbf{A}$ & $82.7 \pm 1.7 \mathbf{A}$ \\
\hline F. oxysporum $(n=10)$ & $2.2 \pm 0.2$ B & $57.6 \pm 4.0 \mathbf{A}$ & $70.1 \pm 3.5$ B \\
\hline F. redolens $(n=8)$ & $2.4 \pm 0.2$ B & $47.7 \pm 4.1$ B & $65.8 \pm 4.3 \mathbf{B}$ \\
\hline $\operatorname{FSSC}(n=8)$ & $0.0 \pm 0.0 \mathrm{C}$ & $10.1 \pm 4.9 \mathrm{C}$ & $0.0 \pm 0.0^{\times} \mathbf{C}$ \\
\hline Control $(n=5)$ & $0.0 \pm 0.0 \mathrm{C}$ & $0.0 \pm 0.0 \mathrm{C}$ & $0.0 \pm 0.0 \mathrm{C}$ \\
\hline$p$-value & $* * *$ & $* * *$ & $* * *$ \\
\hline $\begin{array}{l}\text { Fusarium sp. } \\
\text { Soil Isolates }\end{array}$ & Severity on Roots & Frond Weight Loss (\%) & Root Weight Loss (\%) \\
\hline F. proliferatum $(n=12)$ & $3.5 \pm 0.8 \mathbf{A}$ & $49.3 \pm 14.0 \mathbf{A}$ & $71.0 \pm 13.0 \mathbf{A}$ \\
\hline F. oxysporum $(n=49)$ & $2.3 \pm 1.1 \mathbf{B}$ & $20.9 \pm 14.9$ B & $47.9 \pm 20.3 \mathbf{B}$ \\
\hline Control $(n=4)$ & $0.0 \pm 0.0 \mathrm{C}$ & $0.0 \pm 0.0 \mathrm{C}$ & $0.0 \pm 0.0 \mathrm{C}$ \\
\hline$p$-value & $* * *$ & $* * *$ & $* * *$ \\
\hline
\end{tabular}

Mean \pm standard error of final scores are averaged over the number of isolates for each species. Significance through ANOVA test for weight loss ( ${ }^{* * *}$ significance level at $\left.p<0.001\right)$, all-pairwise comparisons were performed trough LSD (less significant difference) at $99.9 \%$ confidence. Significance through Kruskal-Wallis test for disease severity (*** significance level at $p<0.001$ ), comparisons of mean ranks were performed at $99.9 \%$ confidence. ${ }^{\times}$FSSC root weight loss was evaluated as 0 , because the original value was negative, although there was not significance.

In the case of plant isolates, for $54.5 \%$ of F. proliferatum isolates, more than $75 \%$ of inoculated plants died at the end of the experiment, while this high mortality was induced by only $20 \%$ of $F$. oxysporum isolates and $25 \%$ of F. redolens isolates (Figure 7A). In addition, all F. proliferatum, F. oxysporum and F. redolens isolates produced damages on the asparagus root system. Over $50 \%$ of $F$. proliferatum isolates produced more than $60 \%$ of rot roots, while only $30 \%$ of $F$. oxysporum and $25 \%$ of $F$. redolens isolates were so aggressive (Figure 7B). In the case of soil isolates, $16.7 \%$ of $F$. proliferatum isolates killed more than $50 \%$ of inoculated plants, while none of the F. oxysporum isolates induced mortality to more than $50 \%$ of plants, and $46.9 \%$ of $F$. oxysporum isolates did not kill any plants at the end of the tests (Figure 7C). However, all the isolates produced damages on the asparagus root system. For this symptom, $14.2 \%$ of F. oxysporum and $50 \%$ of F. proliferatum isolates were highly pathogenic, rotting more than $80 \%$ of the root system. The distribution of isolates by their aggressiveness based on root impact was different for both species; all F. proliferatum isolates were moderate to highly pathogenic, while F. oxysporum isolates ranged across all degrees of damages in a normal distribution (Figure 7D). This fact reveals the diversity of F. oxysporum found in soils concerning pathogenicity, a reflection of the versatility and biological heterogeneity for this species [43] (Gordon and Martyn, 1997). F. proliferatum was pathogenic for all cases, even though the studied isolates came from soils, not from plant tissues. 

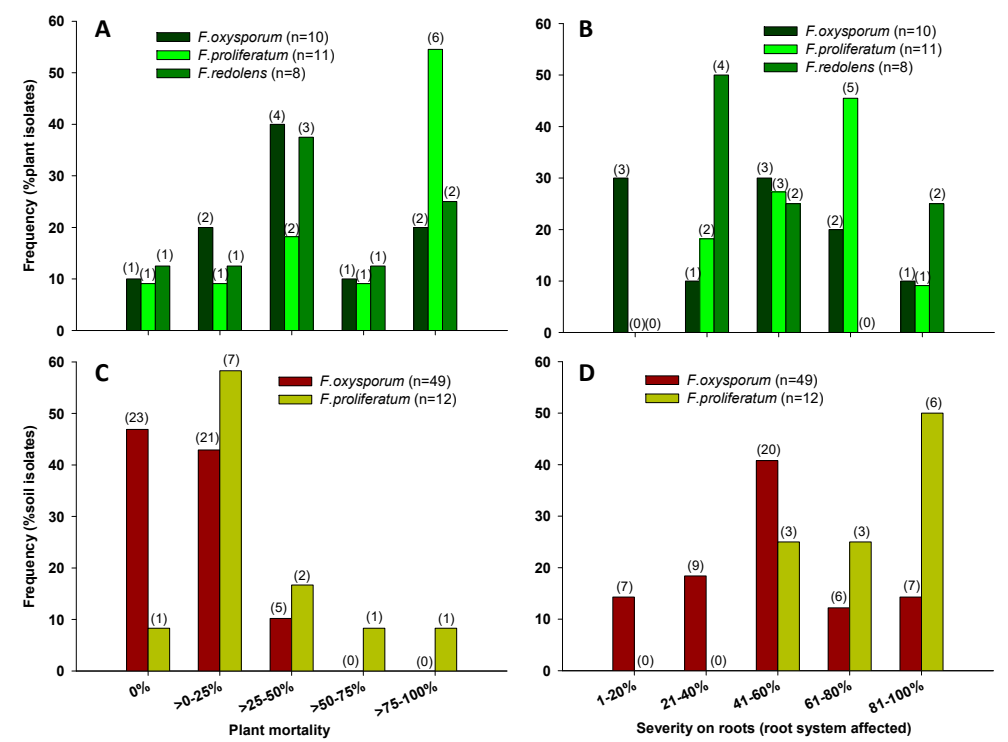

Figure 7. Distribution of plant and soil isolates of the pathogenic Fusarium spp. according to: their mortality to asparagus, respectively $(\mathbf{A}, \mathbf{C})$; and their severity on roots, respectively $(\mathbf{B}, \mathbf{D})$. Values in brackets represent the number of isolates.

With regards to the effect of the inoculations on fronds and roots weights, root damages can explain the decrease of weights for all the species assessed. F. proliferatum produced more damages on roots and reduced asparagus biomass production, in a higher extent than F. oxysporum and F. redolens. Relative root weights decreases were higher than fronds decrease for the three species. The highest impact was observed for F. proliferatum, regardless the origin of the isolates (Table 5).

In addition, F. proliferatum plant isolates presented AUDPC values significantly $(p=0.000)$ higher than the exhibited by F. oxysporum and F. redolens isolates, and these values were significantly higher than those presented by FSSC isolates and the non-inoculated control, which did not present any aerial symptoms (Figure 8).

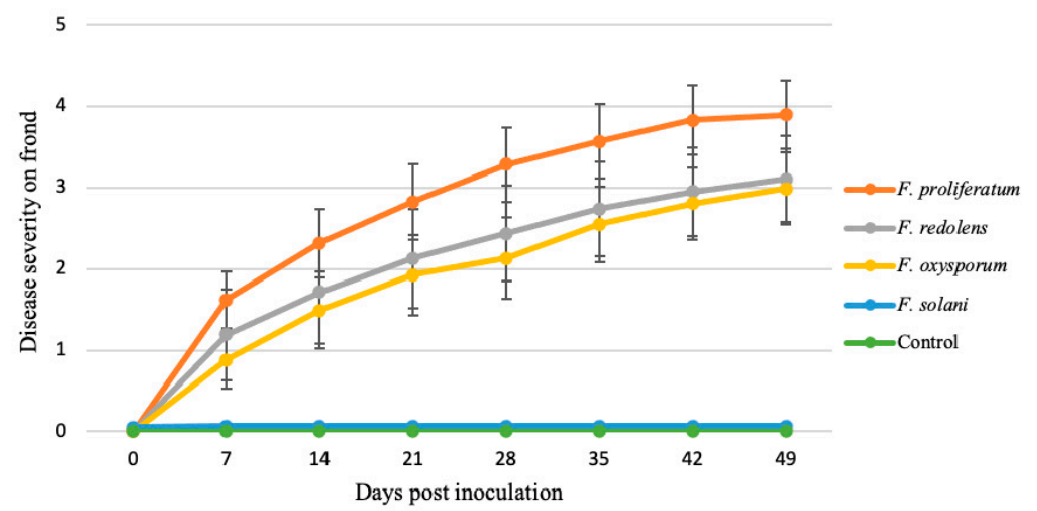

Figure 8. Progress of the severity of symptoms in "Grande F1" asparagus plants inoculated with Fusarium spp. Severity of plant symptoms was measured weekly from one to seven weeks after inoculation on a $0-5$ rating scale. Values are means \pm standard error over the number of isolates for each species. Non-inoculated plants were used as control.

\subsection{Phylogenetic Analysis}

F. oxysporum isolates associated to asparagus diseased plants grouped with isolates from Clades 2 and 3 of FOSC and were classified into phylogenetic species 2 (PS2) according to Laurence et al. [38] with high bootstraps support. Clade 3 was the most numerous group and included isolates from the three regions analyzed. Clade 2 contained isolates from Madrid and Andalusia (Figure 9A). 
The phylogenetic analysis of FFSC isolates from asparagus plants identified all the analyzed isolates as F. proliferatum. They grouped with F. fujikuroi, F. concentricum and F. sacchari into a well-supported group defined as the "Asian Clade" by Kvas et al. [44], and significantly differ from species belonging to the American and African Clade (Figure 9B). F. redolens was the only species of the FRSC detected in asparagus diseased plants. In the phylogenetic analysis, all asparagus isolates grouped with $F$. redolens isolates retrieved from the GenBank and significantly differ from F. hostae and F. spartum species with high bootstrap support (Figure 9C).
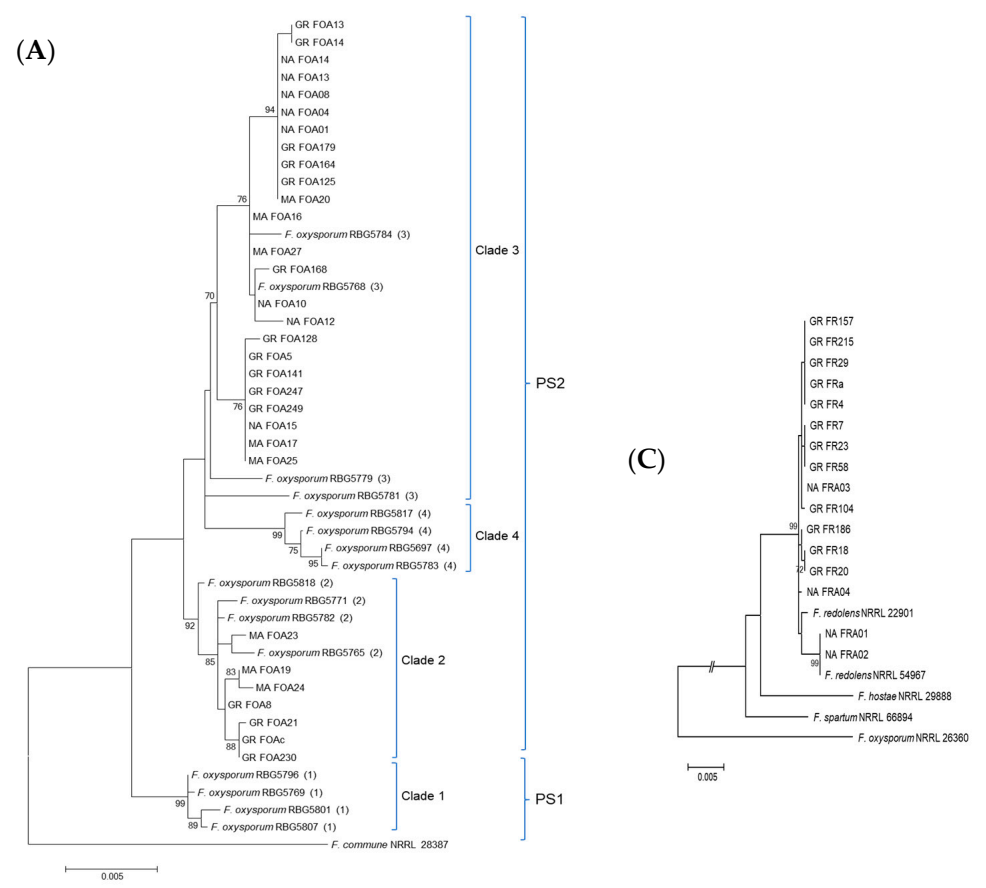

(B)

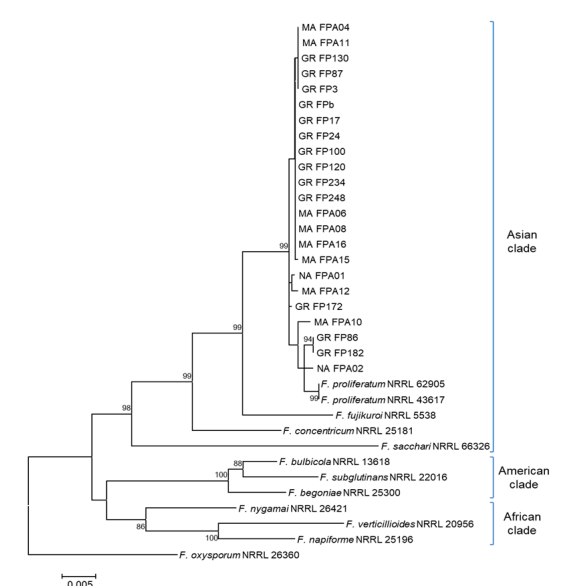

Figure 9. Phylogenetic diversity of Fusarium oxysporum (A), F. proliferatum (B) and F. redolens (C) isolates from asparagus diseased plants of Navarra (NA), Madrid (MA) and Andalusia (GR) production fields, using maximum likelihood analysis of the concatenated translation elongation factor-1a (EF-1 $\alpha)$ (704 base pairs), DNA-directed RNA polymerase II largest (RPB1) (1810 base pairs) and second largest subunit (RPB2) (1596 base pairs) sequence data. Fusarium commune NRRL 28387 and F. oxysporum NRRL26360 were used as outgroups. Support values are above branches and represent bootstrap values of $>70 \%$. For F. oxysporum, the clade designation of $\mathrm{O}^{\prime}$ Donnell et al. (2004) is indicated in parenthesis for isolates retrieved from GenBank, and the phylogenetic species 1 (PS1) and 2 (PS2) correspond to the designation of Laurence et al. (2014). For F. proliferatum, the three clades established by O'Donnell et al. [31] are indicated. 


\subsection{Genetic Diversity}

Haplotype analysis of the pathogenic Fusarium species associated to asparagus diseased plants showed that F. oxysporum was distributed among 14 haplotypes, and F. proliferatum and F. redolens presented nine and eight haplotypes, respectively. The most prevalent haplotypes were present in F. proliferatum and F. oxysporum species. Some F. oxysporum isolates from Navarra, Madrid and Andalusia shared the same haplotype ( $\mathrm{H} 1$ and $\mathrm{H} 2)$, as did some F. proliferatum isolates from Madrid and Andalusia. The distribution of haplotypes was strongly tailed in all analyzed Fusarium species. Unique multilocus haplotypes (singletons) were observed in all species complexes and in the three regions, except for F. redolens that was not detected in plants from prospected fields in Madrid (Figure 10).

A

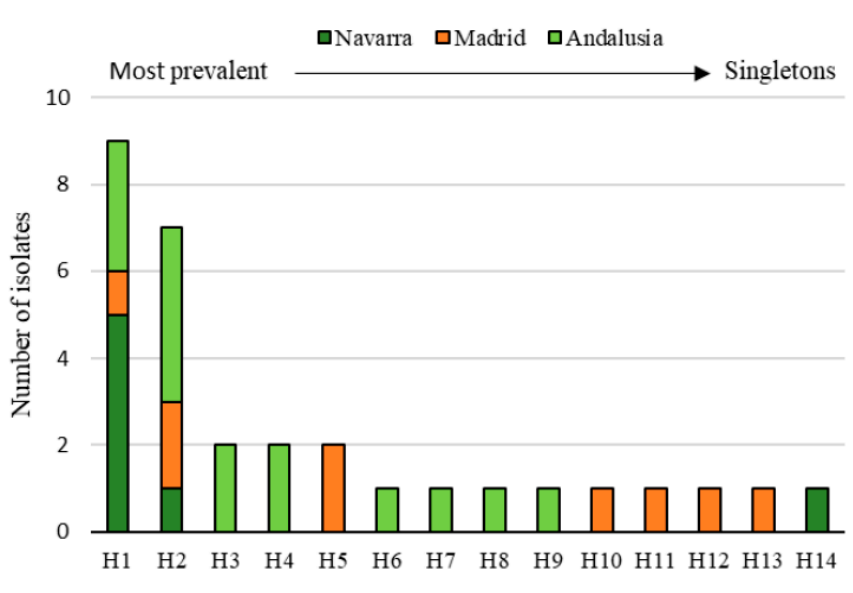

B

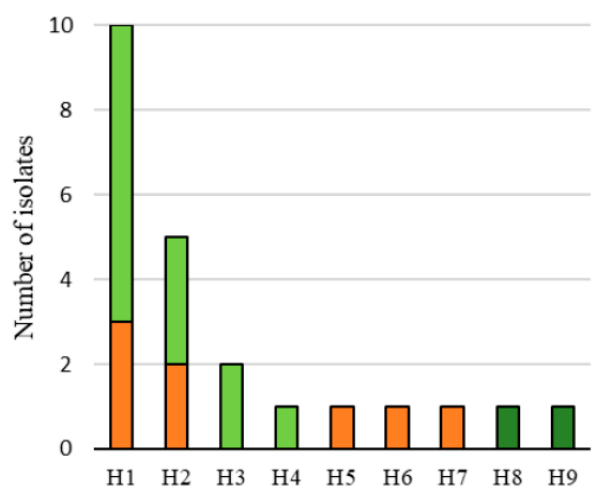

C

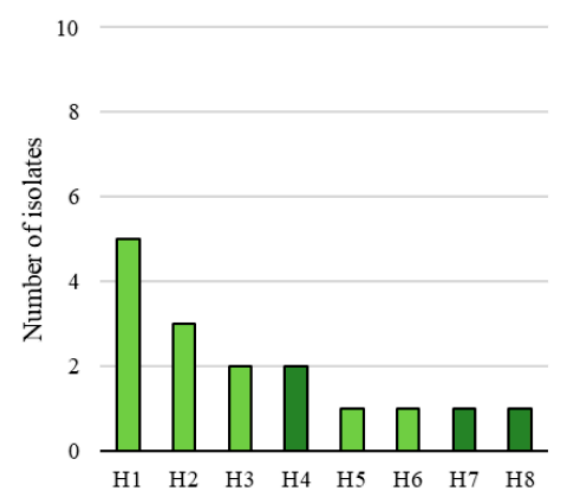

Figure 10. Histogram showing the distribution of isolates from diseased plants from asparagus production fields located in Navarra, Madrid and Andalusia, defined as number of isolates per multilocus haplotype: Fusarium oxysporum (A); F. proliferatum (B); and F. redolens (C).

Genetic diversity analysis showed that F. oxysporum was the species with the highest genetic (haplotypic and nucleotide) diversity. F. proliferatum presented the lowest haplotypic diversity and F. redolens the lowest nucleotide diversity (Table 6). Measures of genetic differentiation were performed for F. oxysporum plant isolates artificially grouped into three populations corresponding to the three prospected areas, to discern differences between populations related to the geographical origin of isolates. No differentiation ( $p$ value of $X^{2}=0.0900$ ) was detected between the $F$. oxysporum populations of Navarra, Madrid and Andalusia production regions (Table S2). 
Table 6. Genetic diversity parameters of Fusarium species associated to asparagus diseased plants estimated by multilocus analysis of $E F-1 \alpha, R P B 1$, and $R P B 2$ partial sequences.

\begin{tabular}{ccccc}
\hline Fusarium spp. & No. of Isolates & No. of Haplotypes & $\begin{array}{c}\text { Haplotype (gene) } \\
\text { Diversity (H) }\end{array}$ & $\begin{array}{c}\text { Nucleotide } \\
\text { Diversity }(\boldsymbol{\pi})\end{array}$ \\
\hline F. oxysporum & 31 & 14 & $0.888 \pm 0.043$ & $0.0044 \pm 0.0004$ \\
F. proliferatum & 23 & 9 & $0.779 \pm 0.074$ & $0.0017 \pm 0.0004$ \\
F. redolens & 16 & 8 & $0.875 \pm 0.059$ & $0.0015 \pm 0.0003$ \\
\hline
\end{tabular}

\section{Discussion}

The main objective of this work was to discern the role of the Fusarium consortium on the Asparagus Decline Syndrome, which severely compromises the current asparagus production in Spain. To achieve this, diseased asparagus plants and soil samples were collected from asparagus fields located in the three main production regions of Spain and studied.

Navarra and Andalusia regions, located in the north and south of the country, respectively, have a long history of asparagus cultivation, and both areas presented a similar pattern of distribution of the main Fusarium species detected in the soil, with the prevalence of F. oxysporum. Analysis of the plant crowns and pathogenicity tests confirmed that the fungus is capable of colonizing the vascular system of the plants, indicating that F. oxysporum f. sp. asparagi is present in diseased plants, as well as in soils, where this forma specialis coexists with F. oxysporum isolates non-pathogenic to asparagus.

This similar pattern of Fusarium species distribution is maintained despite the type of cultivation and asparagus variety used, which is very different between the two areas: while in Andalusia the asparagus is harvested green, when the shoot emerges from the ground and reaches a height of about $20 \mathrm{~cm}$, in Navarra, the plants are grown deeper and under a black plastic cover to prevent the shoot from synthesizing chlorophyll. In this region, the quality of the asparagus is linked to the white color of the fronds, and, for that reason, nighttime harvesting is carried out. Plastic cover allows maintaining the humidity of the soil for much longer, avoiding evapotranspiration and raising the surface temperature, so that the way of cultivation could be favoring the multiplication of the fungus in the soil. Fungal inoculum remains at upper levels in fields dedicated to white asparagus but is more variable, especially at low inoculum level.

Madrid, the central region of asparagus production, presented a different pattern of species distribution probably due to the most recent history of cultivation of this region. The low number of years that asparagus has been grown in the area has not allowed the microbiota associated with the crop to evolve into specialization in the same way as in the other two sampled regions. This can be in concordance with Blok and Bollen [15], who found a negative relationship between the number of asparagus-free years and the inoculum density in soils.

A high correlation $\left(\mathrm{R}^{2}=88 \%\right.$ ) was established between F. oxysporum inoculum density in the soil (CFU/g of soil) and the average temperature of the warmest month of the year. In recent years, the disease has spread through many production areas, warming at the global scale has brought about an evolution of the pathosystems. Recent studies have shown a direct association between mean annual temperature and the abundance of groups of soil-borne fungi, some of them plant pathogens [42]. In the specific case of the asparagus vascular wilt, the warming at the global scale could compromise the crop in many production areas.

The prevalence of $F$. oxysporum compared to other species isolated from asparagus roots (with isolation percentages between 7 and 90 times higher) allows us to assume the greater importance of this species in the early stages of colonization of asparagus plants via the root system. In addition, a positive correlation was found between F. oxysporum f. sp. asparagi inoculum density in the soil and FDI, indicating that the incidence of dead plants was related to the quantity of initial inoculum. In addition, the age of the culture and the average temperature also affects the infection of plants in the field. On the other hand, the greater number of feeder roots affected by F. oxysporum compared to storage roots, pointed to feeder roots as the first point of entry. The propagules would germinate and 
colonize them to later pass to the reserve roots. The main point of entry of the pathogen into the plant is through cuts made to the asparagus shoots during harvest, but entry through the root system should not be underestimated. In this sense, planting soil could be suggested as a source of inoculum for pathogenic Fusarium species. The fact that F. oxysporum was the most prevalent species detected in the soil and in diseased asparagus plants, strongly supports this hypothesis. For that reason, strategies to reduce the level of Fusarium inoculum in pre-planting production soils, such as incorporating organic amendments in biosolarization or biological control [45,46], are highly recommended.

With regard to the pathogenicity of Fusarium species assessed, the Fusarium species found pathogenic (F. oxysporum, F. proliferatum and F. redolens) do not differ from those associated with ADS in previous works $[9,16-19]$. However, it differs from the last information concerning the Spanish asparagus fields sampled 15 years ago, where $F$. solani was shown as a very pathogenic species [17]. Seventy-six FSSC isolates were tested in the present work, and none of them was pathogenic to asparagus. FSSC, currently defined as genus Neocosmospora [47], includes many species that were previously considered F. solani (section Martiella \& Ventricosum, Nelson et al. [29]). This can explain to some extent our differential results. Probably, FSSC species detected in this study (F. solani sensu stricto, F. falciforme, F. tonkinense and F. eumartii) were different from the former F. solani associated to asparagus in the past. Obviously, we did not find those pathogenic $F$. solani isolates in our recent survey. Similar explanation can be found for the works of Lamondia and Elmer [48] and Schreuder et al. [14], who did not find pathogenicity for $F$. solani isolates associated to ADS plants.

F. proliferatum was the most aggressive pathogen of the consortium. Even though F. proliferatum does not produce chlamydospores, thus its survival in soils is supposed to be lower than F. oxysporum or $F$. redolens, the pathogenic ability of F. proliferatum showed on roots was higher than the other species, causing severe necrosis and death of plants. These observations are in concordance with those of Block and Bollen [49] and Tello et al. [20]. F. oxysporum, F. proliferatum and F. solani have been associated to garlic crop in Spain, in cropping areas nearby the asparagus fields [50], which could be linked with the presence of $F$. proliferatum in the environment, as well as the prevalence of pathogenic isolates of the chlamydospore-forming species (F. oxysporum, F. solani) in the soils. The pathogenicity of F. oxysporum f. sp. asparagi and F. redolens was quite similar: both species have common morphological features, and both produce the same type of spores, so their identification might be quite complicated. A precise molecular identification based on multilocus sequence typing via Fusarium MLST and phylogenetic analysis allowed the accurate identification of the species associated to ADS, which is crucial for management.

In addition to being the most abundant species detected in asparagus soils and diseased roots, F. oxysporum exhibited the highest genetic diversity compared to the other two pathogenic species, F. proliferatum and F. redolens, displaying the highest number of haplotypes (gene diversity) and highest number of DNA polymorphisms (nucleotide diversity). This high genetic diversity has been previously observed for this species within FOSC [51] and could imply a mayor difficulty for its control. The ability of this fungus to survive in plant debris and soil for long periods makes its control a big concern, especially when chemical and biological control has proven to be ineffective with high inoculum density in the soil [52].

No genetic differentiation was detected between F. oxysporum f. sp. asparagi isolates in the three asparagus production regions analyzed. In fact, isolates from the three regions grouped in the same phylogenetic group and even shared the same haplotype. This indicates that geographical origin was not strongly correlated with isolate grouping, despite the different history of cultivation and the use of different varieties and culture management in the three Spanish production regions.

\section{Conclusions}

Although eight and thirteen Fusarium species were, respectively, associated to ADS affected asparagus fields and symptomatic roots, our results suggest that the three most pathogenic species in Spanish asparagus fields turned are F. oxysporum f. sp. asparagi, F. proliferatum and F. redolens. 
F. oxysporum was the most prevalent species in all the sampled areas and F. proliferatum the species whose isolates showed the greatest pathogenicity to asparagus.

This work has also revealed a high genetic diversity of F. oxysporum species compared to the other two pathogenic species, although no genetic differentiation related to geographical distribution could be detected.

Our results also reveal epidemiological information about F. oxysporum in asparagus fields, describing the effects of ecological factors such as the temperature on the density of F. oxysporum inoculum in the soil. This result should alert growers of the influence of the climate change on the sanitary status of the asparagus cultures. The effects of some other factors such as water activity remain to be elucidated. We also established a relationship between three crop factors and the disease index values in the field which can be used to monitor fields and will allow farmers to make certain management decisions for new plantations.

Supplementary Materials: The following tables are available online at http://www.mdpi.com/2309-608X/6/4/336/s1, Table S1: Primers used in this study for the amplification of partial sequences of the translation elongation factor- $1 \alpha$ $(E F-1 \alpha)$, and the DNA-directed RNA polymerase II largest (RPB1) and second largest subunit (RPB2) genes of Fusarium spp. isolates from asparagus plants Table S2: Genetic differentiation between Fusarium oxysporum $\mathrm{f}$. sp. asparagi preassigned populations by Chi square test [41].

Author Contributions: Conceptualization, D.P., M.d.C.-G. and N.C.; methodology, D.P., M.d.C.-G. and N.C.; software, E.D.l.L. and J.I.M.-G.; validation, L.G. and A.M.B.; formal analysis, L.G. and J.I.M.-G.; investigation, A.M.B., E.D.1.L. and J.I.M.-G.; resources, D.P. and N.C.; data curation, A.M.B., E.D.1.L. and J.I.M.-G.; writing-original draft preparation, D.P. and N.C.; writing - review and editing, D.P. and N.C.; visualization, M.d.C.-G.; supervision, M.d.C.-G.; project administration, D.P.; funding acquisition, D.P., N.C. and M.d.C.-G. All authors have read and agreed to the published version of the manuscript.

Funding: This work was funded by the "Programa Operativo de Crecimiento Inteligente" INIA and FEDER 2014-2020 (RTA2015-00008-C02-01/02).

Conflicts of Interest: The authors declare no conflict of interest. The funders had no role in the design of the study; in the collection, analyses, or interpretation of data; in the writing of the manuscript, or in the decision to publish the results.

\section{References}

1. FAOSTAT. Food and Agriculture Organization of the United Nations. 2018. Available online: http: //www.fao.org/faostat/es/\#rankings/countries_by_commodity_exports (accessed on 14 May 2020).

2. FEPEX. Federación Española de Asociaciones de Productores Exportadores de Frutas, Hortalizas, Flores y Plantas Vivas. 2019. Available online: https://www.fepex.es/datos-del-sector/exportacion-importacionespa\%C3\%B1ola-frutas-hortalizas (accessed on 14 May 2020).

3. Elena, K. Asparagus Diseases. Eur. J. Plant Sci. Biotechnol. 2007, 1, 76-83.

4. Unterecker, H. Fusarium-Wurzelfäule an Spargeljungpflanzen. Gemüse 1972, 8, 147-148.

5. Smith, A.K.; Peterson, R.L. Examination of primary roots of Asparagus infected by Fusarium. Scanning Electron Microsc. 1983, 3, 1475-1480.

6. Hartung, A.C.; Stephens, C.T. Effects of allelopathic substances produced by asparagus on incidence and severity of asparagus decline due to Fusarium crown rot. J. Chem. Ecol. 1983, 9, 1163-1173. [CrossRef]

7. Nigh, E.L. Stress factors influencing Fusarium infection in asparagus. Acta Hortic. 1990, 271, $315-322$. [CrossRef]

8. Elena, K.; Kranias, L. Fusarium spp. as a cause of crown and root rot of asparagus in Greece. Eur. Mediterr. Plant Prot. Organ. Bull. 1996, 26, 407-411. [CrossRef]

9. Elmer, W.H. Fusarium diseases of asparagus. In Fusarium: Paul E. Nelson Memorial Symposium; Summerell, B.A., Leslie, J.F., Backhouse, D., Bryden, W.L., Burgess, L.W., Eds.; APS Press: St. Paul, MN, USA, 2001; pp. $248-262$.

10. Elmer, W.H. Management of Fusarium crown and root rot of asparagus. Crop Prot. 2015, 73, 2-6. [CrossRef]

11. Schofield, P.E. Asparagus decline and replant problem in New Zealand. N. Z. J. Crop Hortic. Sci. 1991, 19, 213-220. [CrossRef]

12. Cohen, S.I.; Heald, F.D. A wilt and root rot of asparagus caused by Fusarium oxysporum (Schlecht.). Plant Dis. Rep. 1941, 25, 503-509. 
13. Bakel, J.M.M.; Kerstens, J.J.A. Foot rot in asparagus caused by Fusarium oxysporum f. sp. asparagi. Neth. J. Plant Pathol. 1970, 76, 320-325. [CrossRef]

14. Schreuder, W.; Lamprecht, S.C.; Marasas, W.F.O.; Calitz, F.J. Pathogenicity of three Fusarium species associated with asparagus decline in South Africa. Plant Dis. 1995, 79, 177-181. [CrossRef]

15. Blok, W.J.; Bollen, G.J. Inoculum sources of Fusarium oxysporum f. sp. asparagi in asparagus production. Ann. Appl. Biol. 1996, 128, 219-231. [CrossRef]

16. Baayen, R.P.; van den Boogert, P.H.J.F.; Bonants, P.J.M.; Poll, J.T.K.; Blok, W.J.; Waalwijk, C. Fusarium redolens f. sp. asparagi, causal agent of asparagus root rot, crown rot and spear rot. Eur. J. Plant Pathol. 2000, 106, 907-912. [CrossRef]

17. Corpas-Hervias, C.; Melero-Vara, J.M.; Molinero-Ruiz, M.L.; Zurera-Muñoz, C.; Basallote-Ureba, M.J. Characterization of isolates of Fusarium spp. obtained from asparagus in Spain. Plant Dis. 2006, 90, 1441-1451. [CrossRef]

18. Borrego-Benjumea, A.; Basallote-Ureba, M.J.; Melero-Vara, J.M.; Abbasi, P.A. Characterization of Fusarium isolates from asparagus fields in Southwestern Ontario and influence of soil organic amendments on Fusarium crown and root rot. Phytopathology 2014, 104, 403-415. [CrossRef]

19. Papizadeh, M.; van Diepeningen, A.D.; Zamanizadeh, H.R. Fusarium ershadii sp. nov., a Pathogen on Asparagus officinalis and Musa acuminate. Eur. J. Plant Pathol. 2018, 151, 689. [CrossRef]

20. Tello, J.C.; González, M.L.; Lacasa, A. The "Fusariosis" (disease produced by Fusarium spp.) of aspargus in Spain. In Proceedings of the 6th International Asparagus Symposium; Lougheed, E.C., Tiessen, H., Eds.; University of Guelph: Guelph, ON, Canada, 1985; pp. 126-135.

21. Messiaen, C.M.; Casini, R. Recherches sur les fusarioses IV. La sytématique des Fusarium. Ann. Epiphyt. 1968, 19, 387-454.

22. Blok, W.J.; Bollen, G.J. Host specificity and vegetative compatibility of Dutch isolates of Fusarium oxysporum f. sp. asparagi. Can. J. Bot. 1997, 75, 383-393. [CrossRef]

23. Lassaga, S.L.; Camadro, E.L.; Babinec, F.J. Assessing genetic variability for Fusarium resistance in three asparagus populations with an in vitro assay. Euphytica 1998, 103, 131-136. [CrossRef]

24. Fravel, D.; Olivain, C.; Alabouvette, C. Fusarium oxysporum and its biocontrol. New Phytol. 2003, 157, 493-502. [CrossRef]

25. He, C.Y.; Hsiang, T.; Wolyng, D.J. Induction of syfrondsic resistance and pathogen defence responses in Asparagus officinalis inoculated with non-pathogenic strains of Fusarium oxysporum. Plant Pathol. 2002, 51, 225-230. [CrossRef]

26. Kottek, M.; Grieser, J.; Beck, C.; Rudolf, B.; Rubel, F. World Map of the Köppen-Geiger climate classification updated. Meteorol. Z. 2006, 15, 259-263. [CrossRef]

27. Komada, H. Development of a selective medium for quantitative isolation of Fusarium oxysporum from natural soil. Rev. Plant Prot. Res. 1975, 8, 114-125.

28. Tello, J.C.; Vares, F.; Lacasa, A. Análisis de muestras. In Manual de Laboratorio. Diagnóstico de Hongos, Bacterias y Nematodos Fitopatógenos; MAPA: Madrid, Spain, 1991; pp. 9-48.

29. Nelson, P.E.; Toussoun, T.A.; Marasas, W.F.O. Fusarium Species. An Illustrated Manual for Identification; The Pennsylvania State University Press: University Park, PA, USA, 1983.

30. Leslie, J.F.; Summerell, B.A. The Fusarium Laboratory Manual; Blackwell Publishing: Ames, IA, USA, 2006.

31. O’Donnell, K.; Kistler, H.C.; Cigelnik, E.; Ploetz, R.C. Multiple evolutionary origins of the fungus causing Panama disease of banana: Concordant evidence from nuclear and mitochondrial gene genealogies. Proc. Natl. Acad. Sci. USA 1998, 95, 2044-2049. [CrossRef] [PubMed]

32. Shaner, G.; Finney, R.E. The effect of nitrogen fertilization on the expression of slow-mildewing resistance in Knoxwheat. Phytopathology 1977, 67, 1051-1056. [CrossRef]

33. O'Donnell, K.; Sutton, D.A.; Fothergill, A.; McCarthy, D.; Rinaldi, M.G.; Brandt, M.E.; Zhang, N.; Geiser, D.M. Molecular phylogenetic diversity, multilocus haplotype nomenclature, and in vitro antifungal resistance within the Fusarium solani species complex. J. Clin. Microbiol. 2008, 46, 2477-2490. [CrossRef] [PubMed]

34. O'Donnell, K.; Sutton, D.; Rinaldi, M.; Sarver, B.; Balajee, S.; Schroers, H.; Summerbell, R.; Robert, V.; Crous, P.; Zhang, N.; et al. Internet-accessible DNA sequence database for identifying fusaria from human and animal infections. J. Clin. Microbiol. 2010, 48, 3708-3718. [CrossRef] 
35. O'Donnell, K.; Rooney, A.P.; Proctor, R.H.; Brown, D.W.; McCormick, S.P.; Ward, T.J.; Frandsen, R.J.; Lysøe, E.; Rehner, S.A.; Aoki, T.; et al. Phylogenetic analyses of RPB1 and RPB2 support a middle Cretaceous origin for a clade comprising all agriculturally and medically important fusaria. Fungal Genet Biol. 2013, 52, $20-31$.

36. Zhang, N.; O’Donnell, K.; Sutton, D.A.; Nalim, F.A.; Summerbell, R.C.; Padhye, A.A.; Geiser, D.M. Members of the Fusarium solani species complex that cause infections in both humans and plants are common in the environment. J. Clin. Microbiol. 2006, 44, 2186-2190. [CrossRef]

37. O'Donnell, K.; Sutton, D.A.; Rinaldi, M.G.; Magnon, K.C.; Cox, P.A.; Revankar, S.G.; Sanche, S.; Geiser, D.M.; Juba, J.H.; van Burik, J.A.H.; et al. Genetic Diversity of Human Pathogenic Members of the Fusarium oxysporum Complex Inferred from Multilocus DNA Sequence Data and Amplified Fragment Length Polymorphism Analyses: Evidence for the Recent Dispersion of a Geographically Widespread Clonal Lineage and Nosocomial Origin. J. Clin. Microbiol. 2004, 42, 5109-5120.

38. Laurence, M.H.; Summerell, B.A.; Burgess, L.W.; Liew, E.C.Y. Genealogical concordance phylogenetic species recognition in the Fusarium oxysporum species complex. Fungal Biol. 2014, 118, 374-384. [CrossRef] [PubMed]

39. Kimura, M. A simple method for estimating evolutionary rate of base substitutions through comparative studies of nucleotide sequences. J. Mol. Evol. 1980, 16, 111-120. [CrossRef] [PubMed]

40. Librado, P.; Rozas, J. DnaSP v5: A software for comprehensive analysis of DNA polymorphism data. Bioinformatics 2009, 25, 1451-1452. [CrossRef] [PubMed]

41. Hudson, R.R.; Boos, D.D.; Kaplan, N.L. A statistical test for detecting geographic subdivision. Mol. Biol. Evol. 1992, 9, 138-151. [PubMed]

42. Delgado-Baquerizo, M.; Guerra, C.A.; Cano-Díaz, C.; Egidi, E.; Wang, J.T.; Eisenhauer, N.; Singh, B.K.; Maestre, F.T. The proportion of soil-borne pathogens increases with warming at the global scale. Nat. Clim. Change 2020, 10, 550-554. [CrossRef]

43. Gordon, T.R.; Martyn, R.D. The evolutionary biology of Fusarium oxysporum. Annu. Rev. Phytopathol. 1997, 35, 111-128. [CrossRef] [PubMed]

44. Kvas, M.; Marasas, W.F.O.; Wingfield, B.D.; Wingfield, M.J.; Steenkamp, E.T. Diversity and evolution of Fusarium species in the Gibberella fujikuroi complex. Fungal Divers. 2009, 34, 1-21.

45. Blok, W.J.; Lamers, J.G.; Termorshuizen, A.J.; Bollen, G.J. Control of soilborne plant pathogens by incorporating fresh organic amendments followed by tarping. Phytopathology 2000, 90, 253-259. [CrossRef]

46. Borrego-Benjumea, A.M.; Basallote-Ureba, M.J.; Melero-Vara, J.M. Etiology and Management of Asparagus Crown and Root Rot. In Fusarium: Epidemiology, Environmental Sources and Prevention; Ferrari-Ríos, T., Reyes-Ortega, E., Eds.; Novascience Publishers: Hauppauge, NY, USA, 2012; pp. 145-160.

47. Sandoval-Denis, M.; Lombard, L.; Crous, P.W. Back to the roots: A reappraisal of Neocosmospora. Persoonia 2019, 43, 90-1858. [CrossRef]

48. LaMondia, J.A.; Elmer, W.H. Pathogenicity and vegetative compatibility among isolates of Fusarium oxysporum and F. moniliforme colonizing asparagus tissues. Can. J. Bot. 1989, 67, 2420-2424. [CrossRef]

49. Blok, W.J.; Bollen, G.J. Fungi on root and stem bases of asparagus in The Netherlands: Species and pathogenicity. Eur. J. Plant Pathol. 1995, 101, 15-24. [CrossRef]

50. Basallote-Ureba, M.J.; Zurera-Muñoz, C.; Prados-Ligero, A.M.; Melero-Vara, J.M. Nueva enfermedad en el cultivo del ajo ocasionada por Fusarium spp. Phytoma-España 2011, 229, 55-58.

51. O'Donnell, K.; Gueidan, C.; Sink, S.; Johnston, P.R.; Crous, P.W.; Glenn, A.; Riley, R.; Zitomer, N.C.; Colyer, P.; Waalwijk, C.; et al. A two-locus DNA sequence database for typing plant and human pathogens within the Fusarium oxysporum species complex. Fungal Genet Biol. 2009, 46, 936-948. [CrossRef] [PubMed]

52. Reid, T.C.; Kizilkaya, K. Use of fungicides and biological controls in the suppression of Fusarium crown and root rot of asparagus under greenhouse and growth chamber conditions. Plant Dis. 2002, 86, 493-498. [CrossRef] [PubMed]

Publisher's Note: MDPI stays neutral with regard to jurisdictional claims in published maps and institutional affiliations. 